This item was submitted to Loughborough's Institutional Repository (https://dspace.lboro.ac.uk/) by the author and is made available under the following Creative Commons Licence conditions.

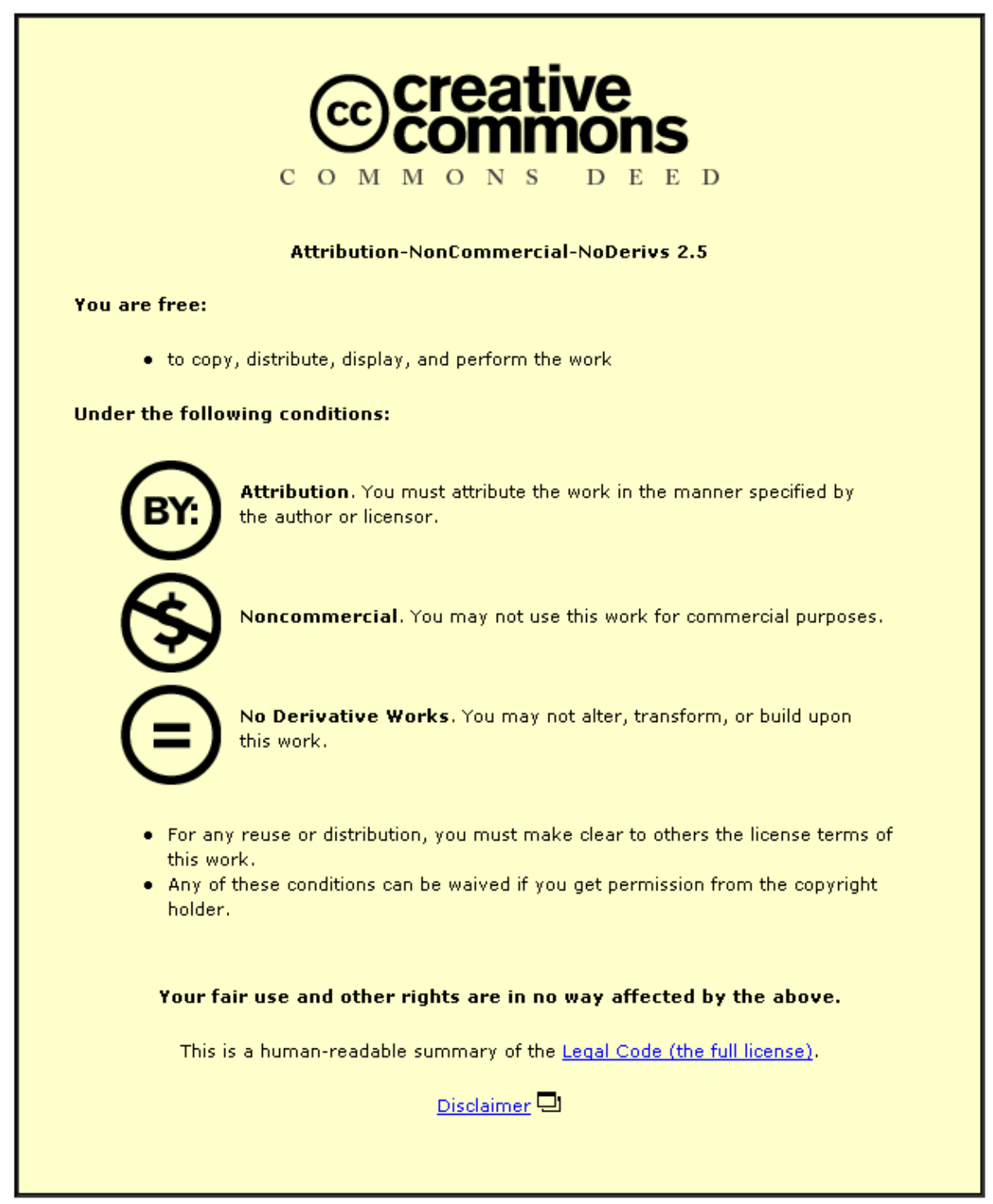

For the full text of this licence, please go to: http://creativecommons.org/licenses/by-nc-nd/2.5/ 


\title{
Dynamic Effects in Capillary Pressure-Saturations Relationships for Two-Phase Flow in 3D Porous Media: Implications of Micro-Heterogeneities
}

\author{
Mahsanam Mirzaei, Diganta Bhusan Das ${ }^{1}$ \\ Department of Engineering Science, Oxford University, Oxford OX1 3PJ, UK
}

Revised Version Submitted for Publication in the Journal:

Chemical Engineering Science

24 November 2006 
Dynamic Effects in Capillary Pressure-Saturations Relationships for Two-Phase Flow in

3D Porous Media: Implications of Micro-Heterogeneities

Mahsanam Mirzaei, Diganta Bhusan Das*

Department of Engineering Science, the University of Oxford, Oxford OX1 3PJ, UK

\section{ABSTRACT}

The capillary pressure-saturation $\left(\mathrm{P}^{\mathrm{c}}-\mathrm{S}\right)$ relationships are essential in characterizing two-phase flow behaviour in porous media. However, these relationships are not unique and depend on the flow dynamics, i.e., steady state or dynamic, among other factors. It has been shown that empirical models describing two-phase flow processes in porous media may be inadequate to account fully for the physics of flow in dynamic conditions. New capillary pressure relationships have been proposed which include an additional term to account for the dependence of capillary pressure on saturation and time derivative of saturation $(\partial S / \partial t)$. This parameter is a capillary damping coefficient, also known as dynamic coefficient $(\tau)$, which establishes the speed at which flow equilibrium is reached. The dependence of $\mathrm{P}^{\mathrm{c}}$-S relationships on $\partial \mathrm{S} / \partial \mathrm{t}$ is called dynamic effects.

In most laboratory experiments for measuring two-phase flow properties, it is implicitly assumed that the sample is homogeneous. However, this is not the case and micro-heterogeneities with their distinct multiphase flow properties may exist within the domain. They affect the dynamics of the multiple fluid phases and saturation distributions in the domain. These issues have been studied individually but the combination of dynamic effects and micro-scale heterogeneities on the $\mathrm{P}^{\mathrm{c}}$-S relationships has not been quantified accurately, particularly in 3D domains. Consequently, there are significant uncertainties on the reported values of $\tau$ in the literature.

In this work, we have carried out a numerical study to investigate how the presence of micro-scale heterogeneities affects the dynamics of dense non-aqueous phase liquid (DNAPL) and water flow in porous domain. The relative significance of the variations in nature, intensity and distribution of micro-scale heterogeneities on dynamic flow conditions are manifested on $\mathrm{P}^{\mathrm{c}}$-S curves which are quantified in terms of the dynamic coefficient, $\tau$. There is a complex interplay of various factors (e.g., dynamic flow conditions, distribution and intensity of micro-heterogeneity, pore size distribution, domain size and geometry and media anisotropy) which affects $\mathrm{P}^{\mathrm{c}}$-S curves. However, our results show that as the intensity of heterogeneity increases the dynamic coefficient at a given saturation increases, provided all other factors remain the same. The effects of domain shapes (cylindrical v/s rectangle), aspect ratios, dimensionality (2D v/s 3D), permeability anisotropy on $\tau$ are also analyzed in order to generalize their effects as far as possible. We envisage that our simulations will minimize some of the inconsistencies on the reported data on $\tau$ in the literature.

KEYWORDS: Two-phase flow; Dynamic effects; Dynamic coefficient; Micro-heterogeneity; Porous media; Capillary pressure; Saturation; Relative permeability; Anisotropy; Aspect Ratio

* Author for Correspondence $\quad$ (Email: $\quad$ Diganta.Das@eng.ox.ac.uk; $\quad$ Tel: $0044 \quad 1865 \quad 283454$ )




\section{Introduction}

A correct description of two-phase flow behaviour in porous media requires the determination of various fluids and media parameters and, the constitutive relationships among capillary pressure $\left(\mathrm{P}^{\mathrm{c}}\right)$, fluid saturation $(\mathrm{S})$ and relative permeability $\left(\mathrm{K}_{\mathrm{r}}\right)$. Traditionally, the $\mathrm{P}^{\mathrm{c}}-\mathrm{S}-\mathrm{K}_{\mathrm{r}}$ relationships have been determined under equilibrium conditions. However, these relationships are not unique and their determination for real soil samples is particularly difficult because of two effects: (i) presence of micro-heterogeneities in the flow domain and (ii) dynamic effects in the $\mathrm{P}^{\mathrm{c}}-\mathrm{S}-\mathrm{K}_{\mathrm{r}}$ relationships. Dependence of the $\mathrm{P}^{\mathrm{c}}-\mathrm{S}-\mathrm{K}_{\mathrm{r}}$ relationships on the time derivative of saturation ( $\partial S / \partial t$ ) is known as the dynamic effect (Hassanizadeh et. al., 2002). While the significances of microscale heterogeneity and dynamic effect on $\mathrm{P}^{\mathrm{c}}-\mathrm{S}-\mathrm{K}_{\mathrm{r}}$ relationships have been studied individually at different scales of observation, the combination of the two effects are not well characterised, particularly in threedimensional (3D) porous domains (realistic domains).

\subsection{Steady State Capillary Pressure Relationship}

Capillary pressure is an essential characteristic of two-phase flow in porous media. In the empirical macroscopic description, $\mathrm{P}^{\mathrm{c}}$ is defined as the difference in average non-wetting and wetting phase pressures and is expressed as a function of wetting phase saturation (S) (Collins, 1961; Scheidegger, 1974, Bear and Verruijt, 1987; Helmig, 1997),

$$
P_{n w}-P_{w}=P^{c, e q u}(S)=f(S)
$$

where $P_{n w}$ and $P_{w}$ are the average pressures of the non-wetting and wetting phases, respectively. This relationship is determined under equilibrium conditions, i.e., when $\partial S / \partial t$ is zero in theory or negligible in practice. Equation (1) is implicitly assumed to account for all effects that influence the equilibrium distribution of fluid(s) in porous media, e.g., fluid properties (e.g., surface tension, viscosity ratio), medium properties (e.g., particle and pore size distributions), wettability of solid surfaces to fluid phases, contact angles, media heterogeneities along with any other parameters. In other words, all effects are lumped into the $\mathrm{P}^{\mathrm{c}}$-S relationship (equation 1), which is then said to characterise the two-phase flow behaviour along with the $\mathrm{K}_{\mathrm{r}}-\mathrm{S}$ relationship. The momentum conservation of the two-phase flow system is generally expressed by an extension of the Darcy’s law as shown in equation (5) (Muskat and Meres, 1936; Leverett 1939; Bear, 1972; Helmig, 1997). In this extension, it is assumed that each fluid phase maintains its own pathways and hence, the effects of any momentum transfer across the fluid/fluid interfaces (e.g., due to molecular interactions) may be ignored in practice (Whitakar, 1986).

\subsection{Dynamic Capillary Pressure Relationship}

However, fluids do not necessarily flow under steady conditions, particularly at smaller time periods when the term $\partial s / \partial t$ may be high. This is supported by a number of experimental and theoretical studies that show that the capillary pressure relationships depend on the flow conditions, i.e. whether it is at steady or unsteady state (Topp et. al., 1967; Vachaud, 1972; Kalaydijan, 1992; Wildenschild, 2001; Barenblatt et. al., 2003; Singh and Mohanty, 2003; Tsakiroglou et. al., 2003; Oung et. al., 2005; Wildenschild et. al., 2005; Das et. al.,2006; Berentsen et. al.., 2006; Bottero et. al.., 2006; Mirzaei et. al.., 2006; Porter et. al., 2006). There are therefore an 
increasing number of authors who suggest that the conventional steady state capillary pressure relationship (equation 1), which assumes that $\mathrm{P}^{\mathrm{c}}$ is a unique function of fluid saturation, may not be sufficient to characterize the two-phase flow behaviour under dynamic conditions (Kalaydijan, 1992; Hassanizadeh and Gray, 1993; Beliaev and Hassanizadeh, 2001; Beliaev and Schotting, 2002; Hassanizadeh et. al., 2002; Dahle et. al., 2005; Hanyga and Seredynska, 2005; O’Carrol et. al., 2005; Oung et. al., 2005; Nordbotten et. al., 2006).

To take into account the dynamic capillary pressure effects on two-phase flow behaviour in porous media, Hassanizadeh and Gray (1993) have proposed a theory, which indicates that the conventional $\mathrm{P}^{\mathrm{c}}$-S relationships (equation 1) can be generalized to include a capillary damping or dynamic coefficient $\tau$ as below:

$$
\left.\left(P^{c, d y n}-P^{c, e q u}\right)\right|_{s}=-\left.\tau(\partial S / \partial t)\right|_{s}
$$

where $P^{c, d y n}$ is the dynamic capillary pressure $\left(P_{n w}^{d y n}-P_{w}^{d y n}\right), P^{c, e q u}$ is the capillary pressure at equilibrium conditions $\left(P_{n w}^{e q u}-P_{w}^{e q u}\right)$, and $\partial S / \partial t$ is the time derivative of saturation, all measured at the same fluid saturation value (S). As evident, equation (2) has the general form of a straight line and, in this form, should pass through the origin. The slope of this linear relationship is the capillary damping coefficient or the dynamic coefficient $(\tau)$. Among other factors, $\tau$ depends on medium and fluid properties, degree of saturation and types of heterogeneity (fractures, layers, etc), if any. If $\tau$ is small, the equivalence between $P^{c, d y n}$ and $P^{c, e q u}$ is established quickly. On the other hand, the necessary time period to reach equilibrium is high for a large value of $\tau$. Thus, the dynamic coefficient behaves as a capillary damping coefficient and indicates the dynamics of the two-phase flow system.

One may argue that the dynamic effects can be studied by the use of Capillary number (Ca), which provides an indication of dynamic effects caused by flow rate at domain boundaries. However, this dynamic rate effect is not the issue here. The dynamic effect investigated in this paper is related to the fact that fluid/fluid interfaces need a finite relaxation time to reach their equilibrium positions at given pressure conditions (Barenblatt et. al., 1990). If the conventional $\mathrm{P}^{\mathrm{c}}$-S relationship is employed to define the dynamic capillary pressure, it is implicitly implied that any disturbance in the system is eliminated instantaneously or within a short period of time. In other words, for the $\mathrm{P}^{\mathrm{c}}$-S- $\mathrm{K}_{\mathrm{r}}$ relationships to be independent of dynamic effects, the two-phase system should not be affected by flow conditions. This is contrary to a number of experimental studies, as discussed before. Therefore, in the last few years, the significance of dynamic effects has been investigated in the contexts of various two-phase flow processes in porous media.

Beliaev and Hassanizadeh (2001) and Beliaev and Schotting (2002) have tested dynamic P ${ }^{\mathrm{c}}$-S relationships and $\tau$ for hysteretic effects, respectively. Theodoropoulou and co-workers (Tsakiroglou et. al., 2003; Tsakiroglou, 2005; Theodoropoulou et. al., 2005) have studied dynamic two-phase flow involving various Newtonian and non-Newtonian fluids. Hassanizadeh et. al. (2002) have reported that the dynamic coefficient varies in the range $3 \times 10^{4}-5 \times 10^{7} \mathrm{kgm}^{-1} \mathrm{~s}^{-1}$. Gielen et. al. (2005) have adopted a dynamic pore-scale network 
model to simulate a 'flow through type cell', often used in the measurement of $\mathrm{P}^{\mathrm{c}}-\mathrm{S}-\mathrm{K}_{\mathrm{r}}$ relationships. They

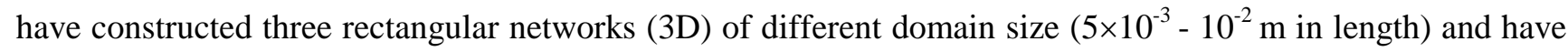
shown that the dynamic coefficients increase with the increase in the averaging network size up to a maximum of $1.2 \times 10^{5} \mathrm{~kg} \mathrm{~m}^{-1} \mathrm{~s}^{-1}$ for the largest pore network they have chosen. Dahle et. al. (2005) have presented a simple 2D pore scale modelling approach based on a bundle of capillary tubes model. Their prime aim has been to demonstrate the pore-scale processes that underlie the dynamic capillary pressure effects. Therefore they adopt a small domain size $\left(\sim 10^{-3} \mathrm{~m}\right)$. As expected, Dahle et. al. obtain small values of $\tau$ which range between $10^{2}-10^{3} \mathrm{~kg} \mathrm{~m}^{-1} \mathrm{~s}^{-1}$. Manthey et. al. (2005) have performed numerical experiments on a number of 2D rectangular homogeneous and heterogeneous domains with dimensions varying between $0.12 \mathrm{~m}$ and $1.0 \mathrm{~m}$. Their simulations correspond to the 'flow through' type cells. Manthey et. al. have shown that the magnitude of $\tau$ depends on the boundary conditions, the domain size and various hydraulic parameters of porous materials. Manthey et. al. ignore the effects of dimensionality (2D v/s 3D) and gravity on the dynamic coefficient and show that the values of $\tau$ may be as large as $10^{9} \mathrm{~kg} \mathrm{~m}^{-1} \mathrm{~s}^{-1}$ for a domain size of $1.0 \mathrm{~m} \times 1.0 \mathrm{~m}$. They also show that for the 2D domains they have chosen, there is negligible difference in the values of $\tau$ in homogeneous (with effective properties) and heterogeneous domain. We show later in our paper that $\tau$ depends strongly on the intensity of heterogeneity, i.e., the amounts of heterogeneities embedded in a porous material. Das and Hassanizadeh (2005) have discussed a number of the above papers (Dahle et. al., 2005; Gielen et. al., 2005; Manthey et. al., 2005). Some of these studies have also been reviewed by O'Carrol et. al. (2005) recently. Based on an inverse modelling approach coupled with an algorithm for optimisation of capillary pressure relationships, O’Carrol et. al. have determined $\tau$ in the range $1 \times 10^{6}-5.7 \times 10^{7} \mathrm{~kg} \mathrm{~m}^{-1} \mathrm{~s}^{-1}$ for a porous material of permeability $\sim 10^{-11} \mathrm{~m}^{2}$. In another recent study, Oung et. al. (2005) have obtained static and dynamic $\mathrm{P}^{\mathrm{c}}$-S curves using a geo-centrifugal facility at $15 \mathrm{~g}$ and $30 \mathrm{~g}$ for a material of permeability $\sim 10^{-12} \mathrm{~m}^{2}$. Based on the difference between these curves, Oung et. al. have obtained $\tau$ in the range $0-600 \mathrm{kgm}^{-1} \mathrm{~s}^{-1}$ at $15 \mathrm{~g}$ and 10-52 $\mathrm{kgm}^{-1} \mathrm{~s}^{-1}$ at 30g. Both O’Carrol et. al. (2005) and Oung et. al. (2005) have used 3D cylindrical porous domains (homogeneous), the same fluids system (Percholoroethylne(PCE)-water) and similar material properties. However, the size of the porous samples and, the experimental and boundary conditions are different in their studies. Consequently both groups of authors obtain distinctly different values of $\tau$. Despite these obvious factors affecting $\tau$, we also believe that the material properties at the smallest scale (e.g. pore and particle size distributions), which affect the bulk material properties (e.g. porosity, permeability, etc), may have influenced the dynamics of two-phase flow studied by O’Carrol et. al. (2005) and Oung et. al. (2005). This is discussed again later when we show that the dynamic capillary pressure depend strongly on the poresize distribution, given by a coefficient in the Brooks-Corey formulation (Brooks and Corey, 1964).

As evident from the above discussion, there is a significant variation on the reported values of $\tau$ in the literatures $\left(0-10^{9} \mathrm{~kg} \mathrm{~m}^{-1} \mathrm{~s}^{-1}\right)$ depending on the size $\left(10^{-3} \mathrm{~m}-1 \mathrm{~m}\right)$ and geometry (2D or 3D) of domains, boundary conditions, porous medium properties (e.g., permeability, entry pressure), fluid properties, types of experiments (e.g., flow through cells or geocentrifuge), type of heterogeneities (e.g., uniform or random micro- 
heterogeneities), etc. Therefore, in principle, one cannot compare directly the values obtained in one study with those obtained in another. One also observes that most of these studies so far have calculated $\tau$ for simplified cases, e.g., homogeneous domain, 2D domain with zero gravity effects, bundle of capillary tubes, etc. This causes a practical difficulty in gauging realistic values of $\tau$ and consequently using them in predicting dynamic capillary pressure relationships in real life problems, which generally involve complex domain characteristics. There is therefore a need to carry out systematic analyses on the sensitivity of the dynamic coefficient to various factors that govern two-phase flow in porous media under realistic conditions (e.g., 3D heterogeneous media), in an attempt to generalise their effects on $\tau$ as far as possible.

We also note that there have been some discussions in the literature on the general form of equation (2) and as to whether the current form shown in equation (2) should include an intercept (C) term that appears in the general equation of a straight line, e.g.,

$$
P^{c, d y n}-P^{c, e q u}=-\tau(\partial S / \partial t)+C
$$

Results of Hassanizadeh et. al. (2002), Gielen et. al. (2005) and, Manthey et. al. (2005) show that the equation (2) does not necessarily pass through the origin of the coordinate system. Dahle et. al. (2005) have adopted a form similar to equation (3) and attempts to correlate the intercept term (C) with other parameters which define the bundle of capillary tubes model that they have developed. Tsakiroglou et. al. (2003) have used a dimensionless form of equation (2) for their studies without the intercept term. Whatever may be the form of equation used for dynamic capillary pressure, one can envisage that the range of validity/applicability of these correlations (i.e., if they can be fitted to a straight line) become increasingly difficult to ascertain as the domain complexity increases. As far as we know, the validity/applicability of equation (2) has not been discussed for two-phase flow in 3D heterogeneous domain in the literature so far.

\subsection{Micro-scale Heterogeneity Effects on Static and Dynamic Capillary Pressure Relationships}

Many geological formations and soils contain small-scale heterogeneities such as micro-scale lenses of fine sand with distinctly different multiphase flow properties from those of the surrounding media. They occur at length scales below those of typical laboratory devices and, have significant effects on two-phase flow behaviour in porous media and, hence, the resulting $\mathrm{P}^{\mathrm{c}}-\mathrm{S}-\mathrm{K}_{\mathrm{r}}$ relationships (Fulcher et. al., 1985; Delshad et. al., 1987; Schwille, 1988; Kueper and Frind, 1991; Illangasekare et. al., 1995a,b; Avraam and Payatakes, 1995; Blom, 1999; Henderson et. al., 2000; Ataie-Ashtiani et. al., 2001, 2002; Das et. al., 2004, 2006). There are some indications that micro-heterogeneities affect the dynamic coefficient, $\tau$ (Hassanizadeh et. al., 2002; Manthey et. al., 2005; Mirzaei et. al., 2006). Most previous studies also show that $\tau$ depends on the domain geometry (2D, 3D rectangle or 3D cylindrical). However, it is not certain if the results obtained for 2D heterogeneous domains (e.g., Manthey et. al., 2005) may be used directly for 3D heterogeneous domains (realistic domains) and if geometry of the domain has any effects on $\tau$-S relationships.

\subsection{Objectives of this Paper}


The main objective of this paper is to characterise the implications of micro-heterogeneities on dynamic twophase flow behaviour in porous materials in terms of the dynamic coefficient $(\tau)$. By 'micro-heterogeneities' we imply fine sand lenses imbedded in coarse background sand. We carry out large numbers of numerical simulations to determine how the interplay of micro-heterogeneities and dynamic flow conditions (given by different pressure boundary conditions) affect the capillary pressure relationships. All simulations are carried out on 3D cylindrical domains, unless otherwise mentioned, for domain dimensions similar to those used in laboratory experiments (12 cm length $\times 10 \mathrm{~cm}$ diameter).

To determine the implications of micro-heterogeneities, we define porous media with different intensities $(\omega)$ and distributions of heterogeneities. The intensity of heterogeneity $(\omega)$ is a non-dimensional parameter given by the ratio of the volume of imbedded fine sand to the total volume of the sample where the background is a coarser sand (Das et. al., 2004, 2006). In this approach, $\omega=0$, 1 define no heterogeneity in the domain, i.e., a homogeneous coarse $(\omega=0)$ or fine $(\omega=1)$ sand domain. Higher is the value of $\omega$ higher is the amount of fine sand in the domain. At $\omega=0.5$, the volume of fine and coarse sand in the domain becomes equal. In cases where $\omega \geq 0.5$, the volume of fine sand is higher than coarse sand and, therefore, the coarse sand gets entrapped in fine sand. In this paper, we attempt to relate the intensity of heterogeneity $(\omega)$ to the dynamic coefficient $(\tau)$ for cases where fine sand is embedded in coarse sand. Although we vary the amounts of imbedded fine sand in the domain, we make sure that each micro-heterogeneity block is the same in size. In effect, we make sure that the size effect of each micro-heterogeneity on $\tau$ is the same and any change on effective static and dynamic capillary pressure relationships on the larger scale is because of the difference in the intensity and distribution of micro-heterogeneities and, not because of the difference in their size.

Recent works have shown that $\tau$ varies non-linearly with fluid saturation (S) (Hassanizadeh et. al., 2002; Manthey et. al., 2005; Bottero et. al., 2006; Mirzaei et. al., 2006). In this paper, we determine the $\tau$-S relationships for 3D heterogeneous domains. Also, based on our results we obtain the $\tau$-S- $\omega$ relationships, in an attempt to generalize the effects of micro-heterogeneities on dynamic capillary pressure relationships. We analyse the implications of micro-heterogeneities on steady and dynamic $\mathrm{P}^{\mathrm{c}}$-S curves. The $\mathrm{K}_{\mathrm{r}}-\mathrm{S}$ curves for various cases are not presented in this paper since they are not used to determine the values of $\tau$. Discussions on $\mathrm{K}_{\mathrm{r}}-\mathrm{S}$ curves in various cases may be found in the literature (e.g., Hassanizadeh et. al., 2002; Singh and Mohanty, 2003; Das et. al., 2004, 2006; Theodoropoulou et. al., 2005).

We also determine how the dynamic coefficient is affected by (i) domain geometries (2D rectangle, 3D rectangle and cylinder) (ii) aspect ratio in 3D cylindrical domain (homogeneous and heterogeneous) and, (iii) anisotropic ratios in permeability in 3D cylindrical domains (homogeneous and heterogeneous). In these cases, we maintain the same boundary conditions and material properties, unless otherwise stated. Our main interests in these simulations are to address some of the uncertainties in the significance of $\tau$ in the literature, as discussed in sections 1.2 and 1.3. 
Further, we discuss briefly if equation (2) is applicable/valid for cases when micro-heterogeneities are present in 3D flow domain. This mainly involves analysing whether the equation (2) maintains the general form of a straight line. With these analyses, we then identify the magnitude of the intercept (C) for 3D heterogeneous cases, as shown in equation (3). However, note that we do not make any attempt to correlate the intercept with various parameters that govern the two-phase flow in porous media. This requires a very high number of simulations and is beyond the scope of this paper.

\section{Descriptions of Numerical Simulations}

\subsection{Governing Model Equations}

The conservation of fluid mass in the two-phase flow system is described by the following equation,

$$
\frac{\partial}{\partial t}\left(\phi \rho_{\gamma} S_{\gamma}\right)+\nabla \cdot\left(\rho_{\gamma} q_{\gamma}\right)=0 \quad \text { for } \gamma \equiv w, n w
$$

where, $\phi[-]$ is porosity, $S_{\gamma}[-]$ is the saturation of corresponding phase, $\rho_{\gamma}\left[\mathrm{Kg} \mathrm{m}^{-3}\right]$ is the fluid density and $q_{\gamma}\left[\mathrm{m}^{3} \mathrm{~s}^{-1}\right]$ is the fluid flux. In addition, the multiphase version of Darcy's equation (equation 5) is used to describe the conservation of fluid momentum in the porous medium.

$$
\bar{q}_{\gamma}=-\frac{K_{r \gamma} K}{\mu_{\gamma}}\left(\nabla . P_{\gamma}+\rho_{\gamma} g \nabla . z\right)
$$

where, $\bar{q}_{\gamma}\left[\mathrm{m}^{3} \mathrm{~s}^{-1}\right]$ is the Darcy flux, $K_{r \gamma}[-]$ is the relative permeability of the corresponding phase, $\nabla . P$ $\left[\mathrm{Nm}^{-3}\right]$ is the driving force, $K\left[\mathrm{~m}^{2}\right]$ is intrinsic permeability of the medium, $\mu_{\gamma}\left[\mathrm{kg} \mathrm{m}^{-1} \mathrm{~s}^{-1}\right]$ is the viscosity of the corresponding fluid phase, and $\nabla . z[-]$ is the upward unit vector.

The average saturation of the wetting and non-wetting phases in the domain are related as below,

$$
S_{w}+S_{n w}=1
$$

The $\mathrm{P}^{\mathrm{c}}$-S relationships are governed by the Brooks-Corey formulations (Brooks and Corey, 1964) as below,

$$
\begin{array}{ll}
S_{e w}=\left(\frac{P^{c}}{P^{d}}\right)^{-\lambda} & \text { for } P^{c} \geq P^{d} \\
S_{e w}=1 & \text { for } P^{c} \leq P^{d} \\
S_{e w}=\left(\frac{S_{w}-S_{r w}}{1-S_{r w}}\right) & \text { for } 0 \leq S_{e w} \leq 1
\end{array}
$$

where, $\mathrm{S}_{\mathrm{ew}}[-]$ the effective wetting phase saturation, $P^{d}\left[\mathrm{Nm}^{-2}\right]$ is entry pressure, $\lambda[-]$ is pore size distribution index and $\mathrm{S}_{\mathrm{rw}}[-]$ is irreducible wetting phase saturation.

The relative permeabilities, $K_{r n}$ and $K_{r n w}$, of the wetting and non-wetting phases in the domain are governed by the following Brooks-Corey-Burdine formulae (Brooks and Corey, 1964), 


$$
K_{r n w}=\left(1-S_{e w}\right)^{2}\left(1-S_{e w}{ }^{(2+\lambda) / \lambda}\right)
$$

\subsection{Averaging Methods for Capillary Pressure Curves}

262

263

264

265

266

267

268

269

270

271

272

273

274

275

276

277 Saturation Weighted Average of $\mathrm{P}^{\mathrm{c}}:\left.P^{c}\right|_{t_{n}}=\left.\left\langle P_{n w}\right\rangle\right|_{t_{n}}-\left.\left\langle P_{w}\right\rangle\right|_{t_{n}}=\frac{\sum_{j=1}^{m}\left(1-S_{w j}\right) P_{n w j}}{\sum_{j=1}^{m}\left(1-S_{w j}\right)}-\frac{\sum_{j=1}^{m} S_{w j} P_{w j}}{\sum_{j=1}^{m} S_{w j}}$

The averaging methods for capillary pressure data are discussed briefly in this section. The main assumption of our simulations/averaging methods is that we do not include any term for the effects of fluid/fluid interfacial area and common lines (see e.g., Murdoch and Hassanizadeh, 2002) on the macroscopic flow behaviour in porous media, as traditionally done for the extended version of Darcy's law for multiphase flow problem under steady-state condition. Theories have been proposed that allow inclusion of these effects on the macroscopic two-phase flow behaviour (e.g., Murdoch and Hassanizadeh, 2002). However, these are not trivial to implement in the type of continuum scale simulations carried out in this paper. It is perhaps more straightforward to include these effects in pore scale models (e.g., Held and Celia, 2001). However we note that our approach is consistent with most studies on dynamic effects at the macro-scale, which do not include effects of the fluid/fluid interfacial and common lines exclusively (Hassanizadeh et. al., 2002; Manthey et. al., 2005; O’Carrol et. al., 2005; Oung et. al., 2005; Berentsen et. al.., 2006; Bottero et. al., 2006).

In our approach, the average capillary pressure and water saturation are calculated after each time step for both steady state and dynamic capillary pressure curves. These are based on the saturation-weighted average of fluid pressures in individual nodes in the domain, as below,

where, $t_{n}[\mathrm{~s}]$ is an arbitrary $n^{\text {th }}$ time step, $\left.\left\langle P_{n w}\right\rangle\right|_{t_{n}}$ and $\left.\left\langle P_{w}\right\rangle\right|_{t_{n}}\left[\mathrm{Nm}^{-2}\right]$ are the volume averaged non-wetting and wetting phase pressure, $P_{n w j}$ and $P_{w j}\left[\mathrm{Nm}^{-2}\right]$ are the non-wetting and wetting phase pressures, and $S_{n w j}$ and $S_{w j}[-]$ are the saturation corresponding to non-wetting or wetting phase pressure at time $t_{n}$ in an arbitrary $j^{\text {th }}$ node where $j=1,2,3, \ldots \mathrm{m}$, m being the total number of nodes in the domain.

At each node of the numerical grid, the saturation and volume of the wetting and non-wetting fluids are related as follows,

$$
\begin{aligned}
& V_{w j}+V_{n w j}=\phi \times V_{j} \\
& S_{w \gamma}=\frac{V_{y j}}{V_{j} \times \phi}, \quad \gamma \equiv w, n w
\end{aligned}
$$

$$
S_{w j}+S_{n w j}=1
$$

where, $V_{w j}, V_{n j}$ and $V_{j}$ are the volumes of the wetting and non-wetting phases in the 3D cell centred around the arbitrary node $j$, respectively. $S_{n w j}$ is the saturation of the non-wetting phase at node $j$. 
Average water saturation in the domain is calculated using an averaging of saturation of individual node in the

whole domain,

Average Saturation: $\left.S_{w}\right|_{t_{n}}=\frac{\left.\sum_{j=1}^{m} S_{w j} V_{j}\right|_{t_{n}}}{\sum_{j=1}^{m} V_{j}}$

294

295

296

297

298

299

300

301

302

303

304

305

306

Based on the averaged water saturations at different time levels, the time derivative of saturation $(\partial s / \partial t)$ is then calculated, which is the slope at a point given by average water saturation and time level on a S-t curve. It is approximated based on a central differencing scheme, as shown below,

The time derivative of saturation: $\left.\frac{\partial S}{\partial t}\right|_{S_{w}, t_{n}}=\frac{\left.S_{w}\right|_{t_{n+1}}-\left.S_{w}\right|_{t_{n-1}}}{t_{n+1}-t_{n-1}}$

where, $\left.S_{w}\right|_{t_{n+1}}$ is the average wetting phase saturation at time step ' $n+1$ ' calculated after the equation (16).

\subsection{Simulator Used}

The two-phase flow simulations have been carried out using the simulator STOMP (ㅁubsurface Transport $\underline{\text { Over }}$ Multiple Phases). The code is developed by the Pacific Northwest National Laboratory, US (www.pnl.gov) and is capable of simulating 11 different modes of multiphase flow and multi-component transport in porous media. In this case, we use the Water-Oil mode to simulate the dynamic and steady state two-phase flow behaviour. STOMP has been successfully validated and applied to model a variety of multiphase flow problems (e.g., Scroth et. al., 1998; Oostrom and White, 1998; Oostrom et. al., 1997; Ataie-Ashtiani et. al., 2001, 2002; Das et. al., 2004, 2006). Ataie-Ashtiani et. al. (2001) have demonstrated the applicability of STOMP to model flow of DNAPL and water in 2D heterogeneous domain by simulating a multi-fluid laboratory experiment described by Kueper and Frind (1989). The spatial discretisation of the governing equations is done based on the standard finite volume method, FVM (Patankar, 1980; Versteeg and Malalasekera, 1995). The discretised equations are described in detail by White and Oostrom (2000). The system of algebraic equations derived through the FVM discretisation of the governing equations and the constitutive equations for $\mathrm{P}^{\mathrm{c}}-\mathrm{S}-\mathrm{K}_{\mathrm{r}}$ relationship involve non-linear terms. These non-linear equations are reduced to linear forms by the application of the Newton-Raphson iterative methods for multiple variables. The temporal discretisation is based on the implicit time stepping method.

\subsection{Descriptions of Porous Media, Fluids Properties and Domains Geometries}

Properties of fine and coarse sand, including intrinsic permeability, porosity, Brooks-Corey-Burdine parameters along with the fluid properties are listed in Table 1. Figure 1 displays a heterogeneous domain including 3D cylindrical column and, 2D and 3D rectangular domains. As discussed later, these domains are used to determine the effects of domain geometries on the dynamic coefficient. The dimensions of these domains are also shown in Figure 1. 


\subsection{Initial and Boundary Conditions for Numerical Simulations}

325

326

327

328

329

330

331

332

333

334

335

336

337

338

339

340

341

342

343

344

345

346

347

348

349

350

351

352

353

354

355

356

357

358

359

There are two main laboratory methods of $\mathrm{P}^{\mathrm{c}}-\mathrm{S}-\mathrm{K}_{\mathrm{r}}$ measurements, namely, the flow-through and pressure-cell experiments (Osoba et. al., 1951; Johnson et. al., 1959; Ataie-Ashtiani et. al., 2002). Our simulations correspond to the pressure cell experiments for determining drainage $\mathrm{P}^{\mathrm{c}}-\mathrm{S}-\mathrm{K}_{\mathrm{r}}$ relationships. In these experiments, the porous sample is made fully saturated with water and placed inside a reservoir filled with a non-wetting phase. In our case we use PCE as the non-wetting phase, a common DNAPL contaminant found in the subsurface. We apply constant pressure for PCE flow and 'no flow' boundary condition (BC) for water at the top of the domain. 'No flow' BCs for both phases are imposed on the side of the domain. At the bottom boundary of the domain, constant pressure for water and 'no flow' BC for PCE is applied. The outflow is defined to be leveled with the top of the domain. This allows only water to drain out and overcomes free downward flow of water. An example BC at the top and bottom of a 3D domain for different cases of dynamic displacement of water by PCE is presented in Table 2. All simulations are carried out in the vertical direction (downward displacement aligned with gravity) and therefore the gravity effects are included in all simulations. However, we do not attempt to synthesize the gravity effects exclusively on the dynamic coefficient in this paper.

\subsection{Quasi-static and Dynamic Two-phase Flow in Porous Media}

A series of dynamic and steady-state downward (along gravity) displacement experiments are simulated involving water as wetting and PCE as non-wetting phase. In all simulations, PCE infiltrates at the top of either 3D cylindrical column or 2D and 3D rectangular domains initially fully saturated with water. The domain is composed of either fine or coarse sand for homogeneous domains. Heterogeneous domains are represented by binary combinations of small fine sand blocks imbedded in background coarse sand.

The procedure for simulating quasi-static displacement is as follows. The initial pressure of PCE is defined to be zero everywhere. Then, the pressure of the non-wetting phase on the boundary of injection is gradually increased. This leads to an increase in average capillary pressure, $\mathrm{P}^{c}$. Once $\mathrm{P}^{c}$ reaches the entry pressure $\left(\mathrm{P}^{d}\right)$ of the background medium, PCE infiltrates the sample and displaces water. The simulation is carried out until steady-state flow conditions are reached, that is, saturation at all grid points does not vary any more with time or this variation is smaller than a tolerance limit $\left(10^{-6}\right.$ in this case). The calculated average saturation and $\mathrm{P}^{\mathrm{c}}$ provide one point of the $\mathrm{P}^{\mathrm{c}}-\mathrm{S}$ curve. Next, the imposed PCE pressure is increased and the simulation is continued until a new steady state is reached. A second point for $\mathrm{P}^{\mathrm{c}}-\mathrm{S}$ curve is thus obtained. This procedure is repeated until a $\mathrm{P}^{\mathrm{c}}$ of $11000 \mathrm{~Pa}$ is reached by which point the sample is deemed to have reached its irreducible wetting phase saturation $\left(S_{i w}\right)$.

For simulating dynamic two-phase flow behaviour, the imposed PCE pressure at the top of domain is increased to a high pressure once and drainage takes place until the domain reaches its $S_{i w}$. We carry out simulations for only drainage. Then, dynamic and steady state $\mathrm{P}^{\mathrm{c}}$-S curves for homogeneous and various heterogeneous domains are prepared. 


\subsection{Determination of Dynamic Coefficient $(\tau)$}

364

Equation (2) shows that if $P^{c, d y n}, P^{c, e q u}$ and time derivative of saturation ( $\left.\partial S / \partial t\right)$ are known at a given saturation value, the damping coefficient $(\tau)$ for a given sample can be determined. To obtain these data simulations corresponding to 'pressure cells', often used for the measurement of $\mathrm{P}^{\mathrm{c}}-\mathrm{S}-\mathrm{K}_{\mathrm{r}}$ curves, are carried out for both steady-state and dynamic two-phase flow in homogeneous and heterogeneous domains. The average values of $P^{c, d y n}, P^{c, e q u}$ and $\partial S / \partial t$ are determined as described in section 2.2. The averaged data are then used to calculate the values of $\left(P^{c, d y n}-P^{c, e q u}\right)$ and $\partial s / \partial t$ for different cases and fitted to a straight line, if possible. The slope of this straight line is then defined as the dynamic coefficient $(\tau)$ for that particular case. The initial and boundary conditions for these simulations are described in section 2.5.

\subsection{Convergence of Numerical Results}

The grid size used in our simulations for 2D and 3D rectangular and 3D cylindrical domains are shown in Table 3. In general, the convergence of the numerical solution is achieved based on a maximum Newton iteration of either 16 or 32, depending on the complexity of the domain type and a tolerance limit of $10^{-6}$. However, we have also checked the convergence of the results through grid refinement. The results showed that the grid refinement has negligible effects on the resultant $\mathrm{P}^{\mathrm{c}}$-S curves. For this test, the normal grid with 24 cells in Z-direction (Table 3) is refined four fold to include 96 cells in the domain whilst the domain size was the same in all cases. The values chosen for $\Delta \Theta$ (nodal spacing in $\Theta$ direction) and $\Delta r$ (nodal spacing in $r$ direction) are kept the same which is a reasonable assumption since the dominant direction of flow is in the Z-direction and not in $\mathrm{r}$ - or $\Theta$ - directions. The $\mathrm{P}^{\mathrm{c}}$-S relationships for the normal (Table 3 ) and the refined grid are then compared. The comparison shows negligible dependence of the $\mathrm{P}^{\mathrm{c}}$-S curves on the chosen grid. The maximum percentage difference of $\sim 1.5 \%$ is found between the $\mathrm{P}^{\mathrm{c}}$-S curves from the two cases. This gives confidence that the results presented in this paper are, in general, independent of the grid size used and the numerical results converge.

\section{Results and Discussion}

\subsection{Validity/Applicability of Equation (2) to Two-phase Flow in 3D Heterogeneous Porous Media}

In this section, we discuss the validity/applicability of equation (2) to dynamic two-phase flow in 3D porous media with micro-scale heterogeneities. For these purposes, we have derived static and dynamic $\mathrm{P}^{\mathrm{c}}$-S curves for various cases as discussed in section 2.6. Figure 2, for example, displays the dynamic and equilibrium capillary pressure curves for homogeneous fine sand (3D cylindrical domain). Plots between the difference in dynamic and equilibrium capillary pressure $\left(P^{c, d y n}-P^{c, e q u}\right)$ versus the time derivative of saturation $(\partial S / \partial t)$ at certain water saturation values are shown in Figures 3(a-c). These figures are then used to determine the dynamic coefficient $(\tau)$ for various homogeneous and heterogeneous domains. Such plots, according to equation (2), should represent a linear relationship passing through the origin. The slopes of these lines give the damping 
coefficient, $\tau$. The results as displayed in Figures 3(a-c) reveal that the linear functions do not necessarily run through the origin and there is an intercept. Further, there is no clear dependence of the intercept term on either the flow conditions or fluid and porous medium properties. The linear equations of the dynamic capillary pressure relationships are directly shown in the figures, which indicate the dynamic coefficient (slope of the straight line) and the intercept.

It seems from our results that the dynamic capillary pressure relationships may not be applicable in certain range of saturations in pressure cells for measurement of two-phase flow properties. In other words, in these cases it may not be possible to fit the data for $\left(P^{c, d y n}-P^{c, e q u}\right)$ and $\partial S / \partial t$ to a straight line with reasonable accuracy. For example, as shown in Figure 3(d), at higher value of water saturation in a homogeneous fine sand media, another relationship in the form of a polynomial function may exist for dynamic capillary pressure. We observe that in general as the average value of water saturation and/or $\partial S$ / $\partial t$ term decrease, the degree of fitness of the data to a straight line increases. In other words, the applicability of equation (2) increases with decrease in average water saturation and/or $\partial S / \partial t$ involving both homogeneous and heterogeneous porous media. This needs to be investigated thoroughly and parameters for polynomial functions for dynamic capillary pressure, if exist, need to be determined and interpreted in terms of two-phase flow parameters in porous media. In this paper, we report values of dynamic coefficient for those ranges of saturations where we obtain reasonable fit of equation (2) to a straight line.

\subsection{Material Properties Effects on Dynamic Coefficient}

In most natural porous media, the material properties, e.g., porosity, permeability, pore size distribution, entry pressure etc., are correlated. It therefore makes more practical sense to synthesise the coupled effects of these parameters on the dynamic coefficient rather than considering the effects of one variable at a time while keeping other variables constant. In this section, we consider the material properties of homogeneous coarse and fine sand as described in Table 1. We have conducted simulations for both dynamic and steady state twophase flow for these material properties. The applied boundary conditions to measure either dynamic or quasistatic capillary pressure are the same for both sand types whilst the displacement time may vary. As expected, a comparison of the $\mathrm{P}^{\mathrm{c}}$-S curves indicates higher irreducible water saturation and capillary pressure at the same saturation in fine sand than those in coarse sand. This is due to higher capillary effect in smaller pores in fine sand compared to coarse sand. This results in higher entrapped (end-point) saturation. Also, time derivative of saturation $(\partial S / \partial t)$ in dynamic flow in fine and coarse sand domain varies for the same boundary pressure.

Calculated dynamic coefficient as a function of the average water saturation for fine and coarse sand domains are shown in Figure 4. The figure shows that the dynamic coefficient is a nonlinear function of the average water saturation and increases as saturation decreases. This is consistent with recent works on determination of dynamic coefficients. However, Figure 4 also indicates that for lower permeability and pore size distribution index $(\lambda)$, as in fine sand, the dynamic coefficient is significantly higher at the same water saturation. This implies that the flow equilibrium is reached slower in these media. The main reason for this is higher capillary 
pressure and lower Darcy velocity for individual fluid phase in fine sand. It takes longer time to drop to the same water saturation in fine sand. Therefore, the time derivative of saturation is smaller in fine sand whilst the difference between dynamic and static capillary pressure curves is almost the same to those in coarse sand (see, e.g., Figure 3(a)). This leads to higher dynamic coefficient values in fine sand domains.

\subsection{Micro-heterogeneity Effects on Dynamic Coefficient}

443 Most laboratory and numerical experiments for the measurements of multiphase flow properties are conducted on homogeneous domains. However, micro-heterogeneities with their distinct multiphase flow behaviour exist in almost every core plug used to conduct these studies. Manthey et. al. (2005) performed numerical two-phase flow displacement in spatially correlated random field of heterogeneity in a 2D rectangular domain. Das et. al. (2006) also investigated effects of random distributions of heterogeneities in 2D rectangular domains on capillary pressure curves without calculating the dynamic coefficient. None of these domain geometries are comparable to real 3D cylindrical core plugs although these studies provide understanding of certain effects. It is therefore not clear if the values of dynamic coefficient obtained for 2D heterogeneous domains may be used directly for 3D heterogeneous cylindrical domains.

To address these issues, we apply a range of PCE pressures to homogeneous and heterogeneous cylindrical domains (3D) with size comparable to those of laboratory core plugs to investigate micro-heterogeneity effects on the dynamic coefficient. The proportion of fine sand lenses embedded in the coarse sand defines the intensity of micro-heterogeneity $(\omega)$, as discussed before in section 1.4. Intensity and distribution of the heterogeneities are varied in different heterogeneous domains. Soil heterogeneities in the form of variations in intrinsic permeabilities $(\mathrm{K})$ and/or entry pressure $\left(P^{d}\right)$ affect the $\mathrm{P}^{\mathrm{c}}$-S curves and hence, the dynamic coefficient. See for example Figures 5(a-b), which display two patterns with different intensities and distributions of micro-heterogeneities within 3D cylindrical domains on $r-\Theta$ and $r-Z$ planes.

In Figures 6(a-d), we show the quasi-static and dynamic $\mathrm{P}^{\mathrm{c}}$-S curves for homogeneous and heterogeneous media for various applied PCE pressures whilst water pressures at domain boundaries remain constant. As expected, the results indicate that the presence of heterogeneity has a significant influence on the effective $\mathrm{P}^{\mathrm{c}}$-S curves compared to the curves for homogeneous porous domains. Figures 6(b-d) show that as the PCE pressure increases, the effective capillary pressure in the domain also increases at a given water saturation value. However, as evident from the dynamic capillary pressure curves, dynamic effects become more prominent at higher PCE pressure (Figure 6(d)). Furthermore, both steady state and dynamic $\mathrm{P}^{\mathrm{c}}$-S curves for heterogeneous media lie between the $\mathrm{P}^{\mathrm{c}}$-S curves for homogeneous coarse and fine sand. Irreducible water saturation in heterogeneous domains is higher than coarse sand and less than that of fine sand. These results are consistent with other studies, which aim to synthesise micro-heterogeneity effects on two-phase flow behaviour (e.g., Ataie-Ashtiani et. al., 2001; Das et. al., 2004, 2006). Most of these studies show that micro-heterogeneities have complex effects on the dynamics of the two-phase flow system. This is discussed below briefly as this enables us to explain our results clearly. 
For any value of intensity of heterogeneity, once the boundary pressure for PCE overcomes the entry pressure of the domain, the PCE starts to infiltrate into the domain. The high density and low viscosity of PCE helps it to flow downwards and drain water out of the domain. Contrast in permeability is important under dynamic conditions because even small variations play a major role in determining the PCE distribution in the heterogeneous sample. The fine sand lenses deflect the downward flow front of PCE because of their low permeability. However, they do not have sufficient lateral extent to serve as a significant barrier to downward flow of PCE. Wherever fine sand blocks are present, they cause local horizontal spreading of PCE at the top of fine sand lenses. However, PCE finds its flow pathway around higher permeability regions and flows downward in coarse background sand. Pools of PCE at the top of fine grained sand and, water trapped in finegrained sand blocks have a lot of interactions with flowing PCE in coarser background sand. Both PCE pools and trapped water behave as an obstruction to PCE flow and water displacement. Because of PCE presence around the fine sand blocks and eventually higher non-wetting phase relative permeability, the relative permeability of water decreases dramatically. This causes water to remain in low permeability layers until the pooled PCE pressure reaches the fine sand entry pressure and invades fine sand region. When this happens, PCE displaces some of the trapped water in fine sand layers. Many small pools in discontinuous lenses are also formed and in some cases it cannot be displaced because of lower relative permeability of wetting phase. These results in a complex PCE distribution across the domain and consequently different effective capillary pressure curves are obtained depending on the intensity and distribution of heterogeneities (Das et. al., 2006). Capillary forces in any low permeability porous medium that PCE can enter trap a certain amount of water as residual water.

As displayed in Figures 6(b-d), the trends (shape) of dynamic capillary pressure curves change at certain water saturations and, the differences between the steady state and dynamic curves become significant. These figures show that the difference becomes more prominent as the applied boundary PCE pressure increases. On the other hand, this effect becomes less pronounced as the proportion of fine sand in the domain (intensity of heterogeneity) increases and completely disappears in case of capillary pressure curves for fine sand. These discrepancies are because of the contributions of gravity/viscous forces, presence of Haines jumps (Haines, 1930; Das et. al., 2006), and in situations when the PCE overcomes the entry pressure of fine sand to displace the entrapped water in fine sand layers. In homogeneous fine sand with low permeability the humps are not seen since there are no heterogeneities.

Using the procedures outlined in section 2.2, we have calculated dynamic coefficients ( $\tau$ ) for homogeneous and heterogeneous domains at different water saturations (S) to quantify the micro-heterogeneity effects on the dynamic coefficient. Our results on $\tau$-S relationships are presented in Figures 6(a-d). In these figures, the intensity of heterogeneity $(\omega)$ increases with increasing the proportion of fine sand lenses embedded in the coarse sand domain. Higher intensity of heterogeneity results in a higher amount of entrapped water in the fine sand blocks at the same boundary conditions. Therefore, the time derivative of saturation for the same 
513 difference in dynamic and static capillary pressure $\left(P^{c, d y n}-P^{c, e q u}\right)$ is less at the same average water saturation

514 in the domain. This results in higher values of dynamic coefficient as the intensity of heterogeneity increases.

516 Our results show that as $\omega$ increases, the difference in the dynamic and equilibrium capillary pressure curves 517 increases. This also yields higher dynamic coefficient values at higher $\omega$ as shown in Figure 7. The figure 518 shows that contrary to homogeneous domains, the inclusion of heterogeneity significantly affects the dynamics 519 of the two-phase flow behaviour in porous media. However, one should not consider the intensity of 520 heterogeneity effect alone since the distribution and location of heterogeneity in the domain with respect to the 521 distance from infiltration also affects the PCE flow (Das et. al., 2004, 2006). For example, consider the 522 distributions of heterogeneities displayed in Figure 5(a-b). They result in different dynamic coefficients at the same saturations for any given PCE pressure.

\subsection{Domain Geometry Effects on Dynamic Coefficient}

As discussed before, it is difficult to make a reliable prediction about the domain geometry effects on the dynamic coefficient based on the reported data in the literature. This is because while there are differences in the domain geometry and size used by various authors, there are also discrepancies in other parameters that affect $\tau$, e.g., material and fluid properties, boundary conditions, etc. To address this issue, we have carried out a systematic study to synthesise the domain geometry effects on $\tau$-S relationships for the same BCs, fluid, and porous medium properties. We have performed simulations for vertically downward flow in both homogeneous and heterogeneous 2D and 3D rectangular and 3D cylindrical domains (Figure 1). Fluid and porous medium properties are the same (Table 1) in all domains. Heterogeneous domains imply binary combination of fine sand and coarse sand. The intensity of heterogeneity in various geometries (e.g., 2D rectangular and 3D domain) is kept the same. This enables us to make a reasonable comparison and explore geometry effects on dynamic coefficient in both homogeneous and heterogeneous media.

As shown in Figure 8(a) and Figure 8(b), the shapes of $\mathrm{P}^{\mathrm{c}}$-S curves from cylindrical domains under dynamic and steady state flow conditions, respectively, are similar to those obtained in 2D and 3D rectangular domains although they are located slightly higher in the dynamic cases. This is due to the dimensionality effect on dynamic fluid flow and saturation distribution in the cylindrical domain, which consequently affects the $\mathrm{P}^{\mathrm{c}}$-S curves.

Figure 9 displays the dynamic saturation versus time for different domain geometries for homogeneous fine and coarse sand. As evident, the time period to reach irreducible wetting-phase saturation increases in fine sand of all geometries. This time duration in coarse sand is less than 6 hours for all domain geometries as shown in the magnified profile in Figure 9. In fine sand, it is found to be more than 1000 hours regardless of domain geometries. This is clearly due to water entrapment in smaller pore spaces and low permeability of find sand. Entrapment of fluid in smaller pores, strong attachment of the wetting fluid to the grain particles and difficulty in displacing the wetting phase due to phase fragmentation are likely to affect saturation distribution in the 
domain. Because of these phenomena, there are more interactions between fluid and porous media in finegrained domains. These affect the effective capillary pressure curves and, hence, the dynamic coefficient.

The $\tau$-S relationships for both homogeneous and heterogeneous 2D rectangular domain and 3D cylindrical columns are discussed here to show if domain geometry has any effect on the dynamic coefficient (Figure 10). Our results show that 3D cylindrical domain results in slightly higher dynamic coefficient values as compared to those values for 2D rectangular domain, at the same saturation. But this difference is negligible in homogeneous domains. This implies that the inconsistencies in dynamic coefficient values are less likely to be caused by geometry if the porous domains are homogeneous and isotropic with respect to material properties (e.g., permeability). However, the differences in the dynamic coefficient values for 2D and 3D heterogeneous domains are more pronounced. This is clearly an effect of the presence of heterogeneities in the domain. For example, the heterogeneities may cause lateral spreading of PCE and entrapment of fluid in the fine sand blocks (Ataie-Ashtiani, et. al., 2001; Das et. al., 2004, 2006). This results in higher dynamic coefficient at the same saturation, as we show in Figure 10.

\subsection{Permeability Anisotropy Ratio Effects on Dynamic Coefficient}

While formulating his law, Darcy defined the permeability to be the same in all directions (Darcy, 1856). However, this is hardly the case ever and anisotropy in permeability frequently occurs in natural porous media in different directions. For example, anisotropy may appear where the soil has deposited in different layers. In many cases, the soil grains have preferred orientations depending on depositional environments, which create anisotropy in the media permeability. Therefore, core samples collected from the field for laboratory experiments are expected to have anisotropy in permeability.

In this section, we aim to characterise the implications of anisotropy in permeability values on the dynamic effects in both homogeneous and heterogeneous porous domains. For this purpose, we use a term anisotropy ratio, which is defined as the ratio of the vertical permeability $\left(\mathrm{K}_{\mathrm{v}}\right)$ to the horizontal permeability $\left(\mathrm{K}_{\mathrm{h}}\right)$ in $3 \mathrm{D}$ cylindrical domain. The ratio gives an indication of the permeability anisotropy, i.e., higher is the ratio higher is the media anisotropy. For our simulations, we vary the ratios by varying vertical permeability while the horizontal permeability is kept constant. Note that in all previous simulations, the anisotropy ratio was 1.0, i.e., the permeability values were the same in both horizontal and vertical directions within homogeneous media. In heterogeneous media, there was a contrast in permeability values of $10^{3}$ between coarse and fine sand. This contrast in permeability is maintained in the heterogeneous domains that are defined for the purpose of this section.

The effects of anisotropic ratio $\left(\mathrm{K}_{\mathrm{v}} / \mathrm{K}_{\mathrm{h}}\right)$ on the dynamic coefficient-saturation ( $\tau$-S) relationships are displayed in Figure 11. We have chosen a homogeneous coarse sand domain (Table 1) and heterogeneous domain with the intensity of heterogeneity of 0.207 to illustrate the effects of permeability anisotropy. As shown in the figure, lower anisotropic ratio (i.e., lower permeability in vertical direction) results in higher dynamic 
coefficient for a given saturation value, especially in heterogeneous domains. This is because the lower permeability in vertical direction behaves as a barrier to the PCE flow and causes it's spreading in the horizontal direction. This also implies slower PCE flow across the domain in vertical direction and larger amount of entrapped water in the micro-heterogeneities in the domain. This results in slow water displacement in the domain in the direction of imposed pressure gradient. Consequently, the equivalence between the static and dynamic $\mathrm{P}^{\mathrm{c}}$-S curves is not established quickly for lower anisotropic ratio. This results in higher values of dynamic coefficient at a given saturation when anisotropy ratio is smaller. When the anisotropy ratio is higher, the reverse is observed, i.e., the dynamic coefficient at any given saturation becomes smaller.

In general, if the permeability anisotropy ratio $\left(\mathrm{K}_{\mathrm{v}} / \mathrm{K}_{\mathrm{h}}\right)$ is small in the direction of fluid flow and/or imposed pressure drop across the flow domain, the dynamic coefficient increases, and vice versa.

\subsection{Domain Aspect Ratio Effects on Dynamic Coefficient}

As discussed before, a number of authors have conducted laboratory and/or numerical two-phase flow experiments in domains of different geometries and size where the material properties and BCs are not necessarily the same. In many cases, the chosen domains have been simplified to address certain questions. It is therefore difficult to make precise idea of the effects of varying domain size on the dynamic coefficient. To address this issue, we have conducted numerical experiments on 3D cylindrical domains (both homogeneous and heterogeneous) with varying aspect ratios (L/D). The effects of domain aspect ratios on the dynamic coefficient are then assessed for three different values by changing the length $(\mathrm{L})$ of the domain while the diameter (D) is kept the same. In the case of the heterogeneous domains, we have kept the same distribution and intensity of micro-heterogeneity. This makes sure that any difference in the results is because of the change in the domain aspect ratio and not because of heterogeneity patterns. We have also kept the same BCs in all cases. In general, BCs have significant effects on the dynamic coefficient (Manthey et. al., 2005). However, we do not make attempt in this paper to address the coupled effects of BCs and aspect ratio on the dynamic coefficients.

$\tau$-S relationships in Figure 12 show that if the aspect ratio is higher (i.e., longer domain), the dynamic coefficient is also higher for both homogeneous and heterogeneous domains at the same effective water saturation. However, the increase in the dynamic coefficient in case of heterogeneous domain is much more dramatic as compared to the homogeneous domains. In the case of longer samples, the distance for DNAPL flow across the domain increases which increases the fluid-solid interactions and fluid residence time in the domain. This results in higher dynamic coefficient values at the same water saturation provided the BCs remain the same. Our results for 3D cylindrical domains qualitatively agree with most previous studies on dynamic coefficient for 2D rectangular domains (flow through type cells), which indicate that the dynamic coefficients increase with increase in domain size (Gielen et. al., 2005; Manthey et. al., 2005). However, we do not compare our results (pressure cells) directly with these studied because of the difference in domain size and geometry, type of flow cell and, boundary conditions used. 


\section{Conclusions}

In this work, we have reported results of our numerical experiments for quasi-static and dynamic two-phase flow in 2D rectangular and 3D cylindrical domains in vertical direction along gravity. The results show that the linear relationship proposed in literature for dynamic capillary pressure is applicable for lower water saturation when $\partial S / \partial t$ is small. However, a polynomial function may be more applicable at higher water saturation when the $\partial s / \partial t$ term is higher. The parameters for these relationships (e.g., dynamic coefficients, os/ot, etc) vary from one case to another depending on many factors, e.g., flow parameters in porous media, domain shape and size, intensity and distribution of heterogeneities, etc. Therefore, the significance of the parameters in the dynamic capillary pressure relations needs to be determined and interpreted carefully in terms of those parameters to apply these relationships in real life scenarios. The simulations carried out in this work provide range of dynamic coefficient values in realistic cases.

Our results show that the non-uniqueness in $\mathrm{P}^{\mathrm{c}}$-S curves and discrepancies in dynamic coefficient are caused not only by dynamic flow condition but also the nature, distribution, and intensity of micro-heterogeneity, pore size distribution, domain size and geometry, and media anisotropy have their own effects. However, there is a complex interplay of different variables. Cylindrical column used to simulate laboratory drainage seems to result in similar $\mathrm{P}^{\mathrm{c}}$-S curves to $2 \mathrm{D}$ and $3 \mathrm{D}$ rectangular domains of equal volume. However, the dynamic coefficient is different from one domain to another, especially in heterogeneous domains. This implies that the dynamics of the flow depend on the geometry of the domain and the $\mathrm{P}^{\mathrm{c}}$-S relationships obtained for one domain geometry may not be used directly for domain of different geometry. For example, in cases where $\mathrm{P}^{\mathrm{c}}-\mathrm{S}$ relationships are upscaled from core scale to larger scales, one should pay particular attention to the shape of the domain geometries in both core and larger scales. Our results also show that the $\mathrm{P}^{\mathrm{c}}$-S curves are subjected to greater dynamic effects in heterogeneous domains.

For micro-heterogeneities of the type used in this study, as the intensity of heterogeneity increases the dynamic coefficient increases. But this dependence is not a linear function and depends also on the distribution of micro-heterogeneity. Values of dynamic coefficient obtained in this study are higher than most previously reported values for the same domain size. However, this is plausible because we consider both 3D and microheterogeneity effects in a dynamic flow condition.

\section{Acknowledgements}

This study has been carried in the framework of the EPSRC (UK) Project GR/S94315/01, "micro-heterogeneity and temperature effects on dynamic capillary pressure-saturation relationships for two-phase flow in porous media”. The EPSRC funding is gratefully acknowledged. Prof. S.M. Hassanizadeh at Utrecht University, the Netherlands is a visiting professor on this project. Prof. Hassanizadeh is acknowledged for many fruitful discussions on the subject. Comments of three anonymous referees, which helped to improve the clarity of the paper, are sincerely acknowledged. 
Ataie-Ashtiani, B., Hassanizadeh, S.M., Oostrom, M., Celia, M.A., White, M.D., 2001. Effective parameters

Ataie-Ashtiani, B., Hassanizadeh, S.M., Celia, M.A., 2002. Effects of heterogeneities on capillary pressure-

Avraam, D. G., Payatakes, A.C., 1995. Flow regimes and relative permeabilities during steady-state two-phase

Barenblatt, G. I., Patzek, T. W., Silin, D. B., 2003. The mathematical model of non-equilibrium effects in water-oil displacement. SPEJ, 84: 409-416.

Barenbaltt, G.I., Entov, V.M., Ryzhik, V.M., 1990. Theory of fluid flows through natural rocks, Kluwer Academic Publishers, Dordrecht, the Netherlands.

Bear, J. and Verruijt, A., 1987. Modelling groundwater flow and pollution. D. Reidel Publ. Co., Dordrecht, the 686

Beliaev, A.Y., Hassanizadeh, S.M., 2001. A theoretical model of hysteresis and dynamic effects in the 689

Beliaev, A.Y., Schotting, R.J., 2002. Analysis of a new model for unsaturated flow in porous media including hysteresis and dynamic effects. Computational geosciences, 5: 345-368. media including non-equilibrium capillary pressure effects. Proceedings of the XVI International Conference on Computational Methods in Water Resources (CMWR), Copenhagen, Denmark.

Blom, S.M.P., 1991. Relative permeability to near-miscible fluids. Delft University of Technology. Delft, The Netherlands, 174 p.

Blunt, M., and King, P., 1991. Relative permeabilities from two- and three-dimensional pore-scale network modeling. J. Transp. Porous Media, 6: 407- 433. 
Bottero, S, Hassanizadeh, S.M., Kleingeld, Bezuijen, A., 2006. Experimental study of dynamic capillary pressure effect in two-phase flow in porous media. Proceedings of the XVI International Conference on Computational Methods in Water Resources (CMWR), Copenhagen, Denmark.

Bourgeat, A., Panfilov, M., 1998. Effective two-phase flow through highly heterogeneous porous media: capillary non-equilibrium effects. Computational Geosciences 2: 191-215.

Brooks, R.H., and Corey, A.T., 1964. Hydraulic properties of porous media. Hydrology Papers. Colorado State University.

Burdine, N. T., 1953. Relative permeability calculations from pore size distribution data. Petroleum Trans. 198: 71-77.

Collins, R.E., 1961. Flow of Fluids through Porous Material, Reinhold Publishing Corporation, New York.

Dahle, H.K., Celia, M.A., Hassanizadeh, S.M., 2005. Bundle-of-tubes model for calculating dynamic effects in the capillary pressure-saturation relationship. Transport in Porous Media, 58(1-2): 5-22.

Darcy, H., 1856. Les Fontaines Publiques de la Ville de Dijon. Paris: Victor Dalmont.

Das, D.B, Hassanizadeh, S.M., Rotter, B.E., Ataie-Ashtiani, B., 2004. A numerical Study of Microheterogeneity Effects on Upscaled Properties of Two-Phase Flow in Porous Media. Transp. Porous Media, 56: 329-350.

Das D. B., Mirzaei, M., Widdows, N., 2006. Non-Uniqueness in Capillary Pressure-Saturation-Relative Permeability Relationships for Two-Phase Flow in Porous Media: Implications of Intensity and Random Distribution of Micro-Heterogeneity. J. Chem. Eng. Science. 61: 6786-6803.

Davidson, J.M., Nielsen, D.R. and Biggar, J.W., 1966. The dependence of soil water uptake and release upon the applied pressure increment. Soil. Sci. Soc. Am. Proc. 30: 298-304.

Delshad, M., Pope, G., Lake, L., 1987. Two- and three-phase relative permeabilities of micellar fluids. SPE Form. Eval., (Sept.), 327- 337.

DiCarlo, D.A. and Blunt, M.J., 2000. Determination of finger shape using the dynamic capillary pressure. Water Resources Research 36(9): 2781-2785.

Fulcher, R. A., Ertekin, T., and Stahl, C. D., 1985, Effect of capillary number and its constituents on two-phase relative permeability curves ,J. Petrol. Tech., (Feb.), 249-260. 
Gielen, A.J.M., Hassanizadeh, S.M., Nordhaug, H.F., Leijnse, A., 2005. Dynamic effects in multiphase flow: a

744

745

746

747

748

749

750

751

752

753

754

755

756

757

758

759

760

761

762

763

764

765

766

767

768

769

770

771

772

773

774

775

776

777

778

779

pore-scale network approach. In Das, D.B. and Hassanizadeh, S.M., 2005. Special issue on: Upscaling multiphase flow in porous media: from pore to core and beyond. Springer, The Netherlands.

Haines, W.B., 1930. "Studies in the physical properties of soil the hysteresis effect in capillary properties and the modes of moisture distribution associated therewith", J. Agricultural Sci., 20, 97-116.

Hanyga, A., Seredynska, M., 2005. A dynamic model of capillary hysteresis in immiscible fluid displacement. Transport in Porous Media, 59:249-265.

Hassanizadeh S.M., Celia, M.A., Dahle, H.K., 2002(a). Dynamic effect in the capillary Pressure- Saturation and its impact on unsaturated flow. Vadose Zone Hydrology, 1: 38-57.

Hassanizadeh, S.M., Celia, M.A., Dahle, H.K., 2002(b). Dynamic effect in the capillary pressure - saturation relationship and its impact on unsaturated flow. Agricultural Sciences, 7(2): 69-71.

Hassanizadeh, S.M., Gray, W.G., 1993(a). Thermodynamic basis of capillary pressure in porous media. Water Resources Research 29(10): 3389-3405.

Hassanizadeh, S.M., Gray, W.G., 1993(b). Toward an improved description of the physics of two-phase flow. Adv. Water Resources, 16: 53-67.

Helmig, R., 1997. Multiphase flow and transport processes in the subsurface. Springer, 367 P.

Henderson, G.D., Danesh, A., Al-kharusi, B., Tehrani, D., 2000. Generating reliable gas condensate relative permeability data used to develop a correlation with capillary number. J. Pet. Sci Eng. 25, 79.

Hilfer, R., 1996. Transport and Relaxation Phenomena in Porous Media. Adv. Chem. Phys., XCII: 299 - 424.

Illangasekare, T.H., Yates, T.H., Armbruster, E.J., 1995. Effects of heterogeneity on transport and entrapment of noneqeous phase waste products in aquifers: an experimental study, ASCE J. of Env. Eng., 121(8): 572-579.

Illangasekare, T.H., Ramsey, J.L., Jensen, K.H., Butts, M., 1995. Experimental study pf movement and distribution of dense organic contaminants in heterogeneous aquifers, J. of Contaminant Hydrology, 20: 1-25.

Johnson, E.F., Bossier, D.P., Naumann, V.O., 1959. Calculation of Relative Permeability from Displacement Experiments. Trans.AIME., 370,216. 
Kalaydjian, F. 1992. Dynamic capillary pressure curve for water/oil displacement in porous media: theory vs. experiment. SPE Paper 24813. SPE Conference. Washington, DC. USA.

King, P.R., 1989. The Use of Renormalization for Calculating Effective Permeability. Transp. Porous Media, 4: 37-58.

786

787

Kueper, B.H., Frind, E.O., 1991. Two-phase flow in heterogeneous porous media 1. Model development. Water Resources Rs. 27: 1049-1057.

789

790

791

Kueper, B.H., Frind, E.O.,1989. An overview of immiscible fingering in porous media. J. Contam. Hydrol. 5:

792

793

794

Kueper, B.H, McWhorter, D.B., 1991. The behavior of dense, nonaqueous phase liquids in fractured clay and 795

Leverett, M. C., 1939. Flow of oil-water mixtures through unconsolidated sands. Trans. AIME 132:149

797

Manthey, S., Hassanizadeh, S.M., Helmig, R., 2005. Macro-scale dynamic effects in homogeneous and 800 heterogeneous porous media, Transp. Porous Media, 58(1-2): 121-145.

rock. Ground Water. 29(5): 716-728.

804

Muskat, M., Mears, M., 1936. The flow of heterogeneous fluids through porous media, Physics.7 (346-363).

806

807

Nordbotten, J.M., Celia, M.A., Dahle, H.K., Hassanizadeh, S.M., 2006. On the definition of macro-scale 808 pressure for multiphase flow in porous media. Proceedings of the XVI International Conference on Computational Methods in Water Resources (CMWR), Copenhagen, Denmark.

810

O'Carroll, D.M., Phelan, T. J., Abriola, L.M., 2005. Exploring dynamic effects in capillary pressure in multistep outflow experiments, Water Resour. Res., 41, W11419, doi: 10.1029/2005WR004010.

Oostrom, M., Hofstee, C., Dane, J.H., 1997. Light nonaqueous-phase liquid movement in a variably saturated sand. Soil Science Society of America Journal, 61, 1547-1554. 
Oostrom, M., Lenhard, J., 1998. Comparison of relative permeability-saturation-pressure parametric models for infiltration and redistribution of a light nonaqueous phase liquid in sandy porous media. Advances in Water Resources, 21:145-157.

Osoba, J.S., Richardson, J.J., Kerver, J.K., Hafford, J.A., Blair, P.M., 1951. Laboratory Measurements of

Oung, O., Hassanizadeh, S.M., Bezuijen, A., 2005. Two-phase flow experiments in a geocentrifuge and the significance of dynamic capillary pressure effect. J. Porous Media, vol. 8(3): 247-257.

Patankar, S.V., 1980. Numerical heat transfer and fluid flow. New York: Hemisphere, 197p

Porter, M.L., Schaap, M.G., Wildenschild, D., 2006. Capillary pressure-Saturation Curves: Towards

Simulating Dynamic Effects with the Lattice-Boltzman Method. Proceedings of the XVI International

Conference on Computational Methods in Water Resources (CMWR), Copenhagen, Denmark.

Stauffer, F., 1978. Time dependence of the relationship between capillary pressure, water content and conductivity during drainage of porous media. In proceedings of the IAHR conference on scale effects in porous media, Thessaloniki, Greece.

Scheidegger, A.E., 1974. Physics of Flow Through Porous Media. Toronto, University of Toronto Press.

Schwille, F., 1988. Dense Chlorinated Solvents in Porous and Fractured Media: Model Experiments. ed.

Chelsea, MI, Lewis Publishers, Inc. 146 pp.

Singh, M., Mohanty, K.K., 2003. Dynamic modelling of drainage through three-dimensional porous materials. Chemical Engineering Science, 58: 1-18.

Theodoropoulou, M.A., Sygouni, V., Karoutsos, V., Tsakiroglou, C.D., 2005. Relative permeability and capillary pressure functions of porous media as related to the displacement growth pattern International Journal of Multiphase Flow, Volume 31, Issues 10-11: 1155-1180

Theodoropoulou, M.A., Karoutsos, V, Tsakiroglou, C., 2001. Investigation of the contamination of fractured formations by non-Newtonian oil pollutants, J. Environ. Forensics 2:321-334

Tsakiroglou, C.D., Theodoropoulou, M.A., Karoutsos, V., 2003. Nonequilibrium Capillary Pressure and 
Tsakiroglou, C.D., 2004. Correlation of the two-phase flow coefficients of porous media with the rheology of shear-thinning fluids, J. Non-Newtonian Fluid Mech, 117:1-23.

857

858 Topp, G.C., Klute, A., Peters, D.B., 1967. Comparison of water content-pressure head obtained by equilibrium, steady-state and unsteady state methods. Soil Sci. Amer. Proc. 31: 312-314.

860

861 Vachaud, G., Vauclin, M., Wakil, M., 1972. A study of the uniqueness of the soil moisture characteristic during desorption by vertical drainage, Soil Sci. Soc. Amer. Proc., 36, 531-532.

863

864

Versteeg, H. K.; Malalasekera, W., 1995. An introduction to computational fluid dynamics - the finite volume 865 method. London, Longman Scientific and Technical, 257p

Wildenschild, D., Hopmans, J. W., Simunek, J., 2001., Flow rate dependence of soil hydraulic characteristics, 868 Soil Sci. Soc. Amer. J., 65, 35-48.

869

870 Wildenschild, D., Hopmans, J.W., Rivers, M.L., Kent, A.J.R., 2005. Quantitative analysis of flow processes in 871 a sand using synchrotron x-ray microtomography. Vadose Zone Journal 4:112-126.

872

873 Whitaker, S., 1986. Flow in Porous Media II: the Governing Equations for Immiscible Two-Phase Flow.

874 Transp. Porous Media, 1, 105.

875

876 White, M.D., Oostrom, M., Lenhard, R.J., 1995. Modelling fluid-flow and transport in variably saturated 877 porous media with STOMP simulator: 1.Non-volatile 3-phase model description. Advances in Water 878 Resources, 18(6), 353-364.

879

880 www.pnl.gov 


\section{$\underline{\text { List of tables }}$}

Table-1: Model parameters used in simulation (adopted from Kueper and Frind, 1989; Ataie-Ashtiani et. al., 2001; Das et. al., 2004, 2006)

Table-2: Boundary condition for different dynamic drainage displacement cases for cylindrical domain sand sample.

Table-3: Number of nodes and nodal spacing for different domain geometries 


\section{$\underline{\text { List of figures }}$}

Figure 1. Representative domains geometry and dimensions used in simulations.

Figure 2. Steady-State and Dynamic Capillary Pressure Curves for Fine Sand Model.

Figure 3(a-d). Dynamic coefficient for (a) fine sand, (b) coarse sand, (c) heterogeneous sample with intensity of heterogeneity ( $\omega$ ) of 0.207 , (d) fine sand at high saturations in cylindrical domain.

Figure 4. Pore size distribution $(\lambda)$ and permeability $(K)$ effects on dynamic coefficient $(\tau)$ value in a homogeneous cylindrical column consisting of either fine or coarse sand.

Figure 5(a-b). 3D cylindrical domains for two intensities $(\omega)$ and distributions of micro-heterogeneities in the form of sub-sample scale fine sand blocks (dark grey regions) embedded in coarse sand background: (a) $\omega=0.207$, and (b) $\omega=0.372$.

Figure 6(a-d). Micro-Heterogeneity effects on (a) Steady-State and, Dynamic $\mathrm{P}^{\mathrm{c}}$-S curves for PCE pressure of (b) $109316 \mathrm{~Pa}$, (c) $112513 \mathrm{~Pa}$, and (d) $122903 \mathrm{~Pa}$ applied at the Top of a cylindrical domain. All results are for downward flow of PCE in cylindrical domains (3D).

Figure 7. The effect of intensity of heterogeneity $(\omega)$ on dynamic coefficient $(\tau)$.

Figure 8. (a) Effective dynamic capillary pressure curves for coarse and fine sand domains and (b) effective steady-state capillary pressure curves for coarse sand in different domain geometries.

Figure 9. Saturation profile for coarse and fine sand considering different domain geometries under dynamic flow condition.

Figure 10. Domain geometry effect on dynamic coefficient; geometry slightly affects dynamic coefficient $(\tau)$ for flow in homogeneous coarse sand and heterogeneous domains for the geometries and dimensions shown in Fig. 1.

Figure 11. Effect of permeability anisotropy on dynamic coefficient for a homogeneous and heterogeneous domain with intensity of heterogeneity $(\omega)=0.207$.

Figure 12. Effect of aspect ratio on dynamic coefficient for homogeneous and heterogeneous domains with intensity of heterogeneity $(\omega)=0.331$. Aspect ratio for cylindrical domain has been defined as Length/Diameter (L/D). 
Table-1: Model parameters used in simulation (adopted from Kueper and Frind, 1989; Ataie-Ashtiani et. al., 2001; Das et. al., 2004, 2006)

\begin{tabular}{lcccc}
\hline Property & Coarse Sand & Fine Sand & Water & PCE (DNAPL) \\
\hline Permeability, $K\left(\mathrm{~m}^{2}\right)$ & $5 \times 10^{-9}$ & $5 \times 10^{-12}$ & - & - \\
Porosity, $\phi(-)$ & 0.40 & 0.40 & - & - \\
Displacement Pressure, $\mathrm{P}^{\mathrm{d}}\left(\mathrm{Nm}^{-2}\right)$ & 370 & 1325 & - & - \\
Pore size distribution index, $\lambda(-)$ & 3.86 & 2.49 & - & - \\
Irreducible Water Saturation, $S_{i w}(-)$ & 0.078 & 0.098 & - & - \\
Density, $\rho\left(\mathrm{kgm}^{-3}\right)$ & 2630 & 2650 & 1000 & 1630 \\
Viscosity, $\mu\left(\mathrm{kgm}^{-1} \mathrm{~s}^{-1}\right)$ & - & - & $1 \times 10^{-3}$ & $0.9 \times 10^{-3}$ \\
Surface Tension, $\sigma\left(\mathrm{Nm}^{-1}\right)$ & - & - & $0.072^{a}$ & $0.035^{b}$ \\
\hline
\end{tabular}
a- Water-air system
b- PCE-water system 
Table-2: Boundary conditions for different dynamic drainage cases for sand sample in cylindrical domain in a pressure cell used for the measurement of two-phase flow properties. Dirichlet BCs of pressure are imposed for DNAPL at the Top and water at the Bottom. Zero flux BCs are imposed for water at the Top and DNAPL at the bottom. This allows only DNAPL infiltration at the Top and water outflow at the Bottom of the pressure cell.

\begin{tabular}{|c|c|c|c|c|c|}
\hline & & Top Boundary & \multirow{6}{*}{$\begin{array}{l}\text { Zero Flux } \\
\text { Water }\end{array}$} & Bottom Boundary & \multirow{6}{*}{$\begin{array}{c}\text { Zero Flux } \\
\text { DNAPL }\end{array}$} \\
\hline Displacement case & $\begin{array}{c}\text { Time Duration } \\
\text { (hr) }\end{array}$ & $\begin{array}{l}\text { Dirichlet DANPL } \\
\text { Pressure }(\mathrm{Pa})\end{array}$ & & $\begin{array}{l}\text { Dirichlet water } \\
\text { Pressure }(\mathrm{Pa})\end{array}$ & \\
\hline Dynamic case-1 & 5.6 & 109316 & & 102510 & \\
\hline Dynamic case-2 & 3.9 & 112513 & & 102510 & \\
\hline Dynamic case- 3 & 2.8 & 117308 & & 102510 & \\
\hline Dynamic case- 4 & 2.1 & 122903 & & 102510 & \\
\hline
\end{tabular}


Table-3: Number of nodes and nodal spacing for different domain geometries

\begin{tabular}{|c|c|c|c|c|c|}
\cline { 2 - 6 } \multicolumn{1}{c|}{} & \multicolumn{5}{c|}{ Number of nodes $\times$ Nodal Spacing } \\
\hline $\begin{array}{c}\text { Domain } \\
\text { geometry }\end{array}$ & $\begin{array}{c}N \times \Delta r \\
(\mathrm{~cm})\end{array}$ & $\begin{array}{c}N \times \Delta \Theta \\
(\text { degree })\end{array}$ & $\begin{array}{c}N \times \Delta X \\
(\mathrm{~cm})\end{array}$ & $\begin{array}{c}N \times \Delta Y \\
(\mathrm{~cm})\end{array}$ & $\begin{array}{c}N \times \Delta Z \\
(\mathrm{~cm})\end{array}$ \\
\hline 2D Rectangular & - & - & $8 \times 1.25$ & $1 \times 7.92$ & $1 \times 0.05,24 \times 0.5,1 \times 0.05$ \\
\hline 3D Rectangular & - & - & $8 \times 1.25$ & $8 \times 0.988$ & $1 \times 0.05,24 \times 0.5,1 \times 0.05$ \\
\hline 3D Cylindrical & $4 \times 1.25$ & $4 \times 90$ & - & - & $1 \times 0.05,24 \times 0.5,1 \times 0.05$ \\
\hline
\end{tabular}

$N$ : Number of nodes

$\Delta r$ : Nodal Spacing in $r$ direction for cylindrical domain

$\Delta \Theta$ : Nodal spacing in $\Theta$ direction for cylindrical domain

$\Delta X$ : Nodal spacing in $\mathrm{X}$ direction for rectangular domain (2D or 3D)

$\Delta Y$ : Nodal spacing in $\mathrm{Y}$ direction for rectangular domain (2D or 3D)

$\Delta Z$ : Nodal spacing in $Z$ direction for rectangular domain (2D or 3D) 

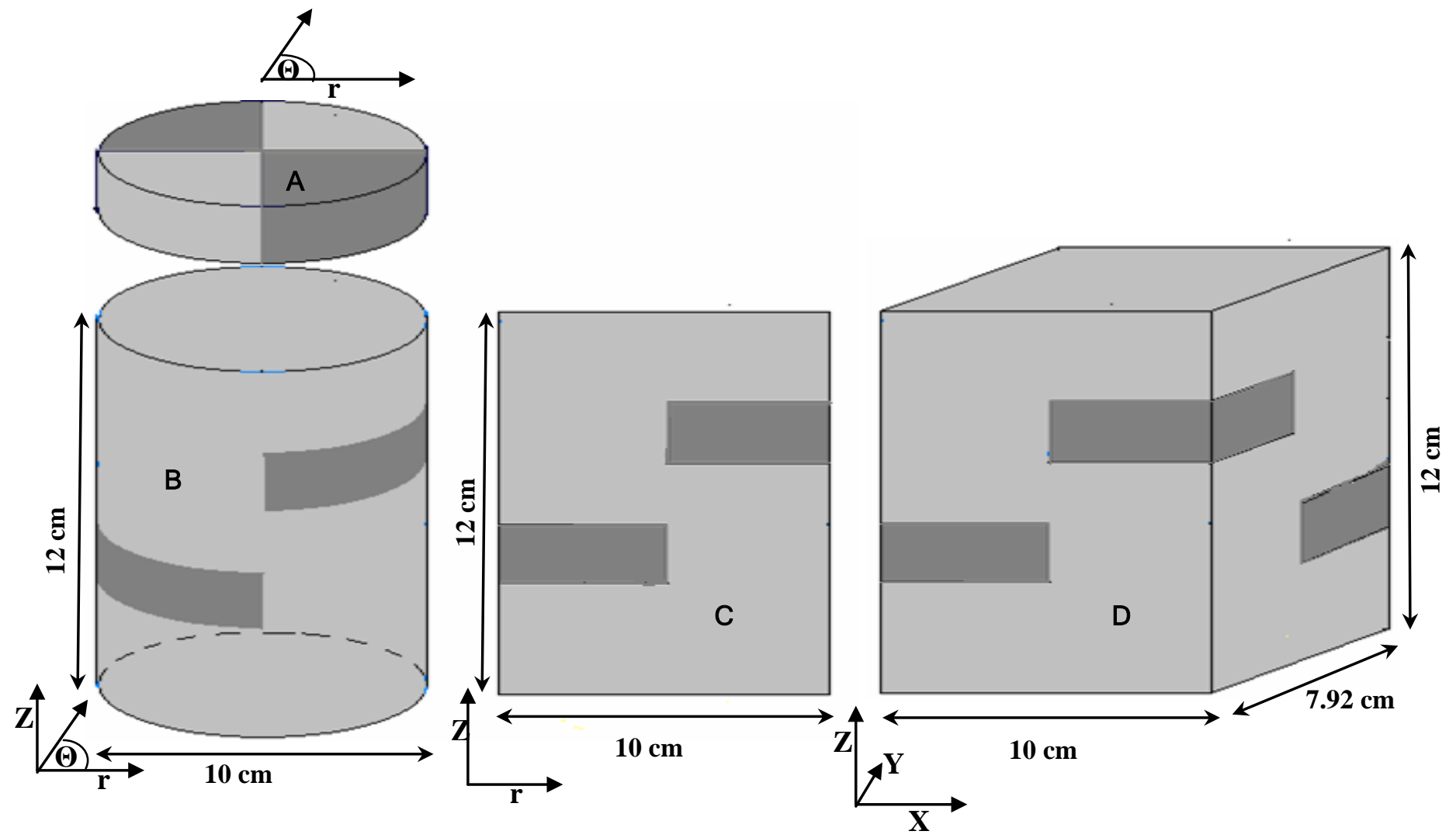

Figure 1. Representative domains used in simulations: (A) $\mathrm{r}-\Theta$ section of heterogeneity distribution, (B) 3D cylindrical domain geometry, (C) 2D (r-Z) section of heterogeneity in a cylindrical domain, (D) 3D rectangular domain. 


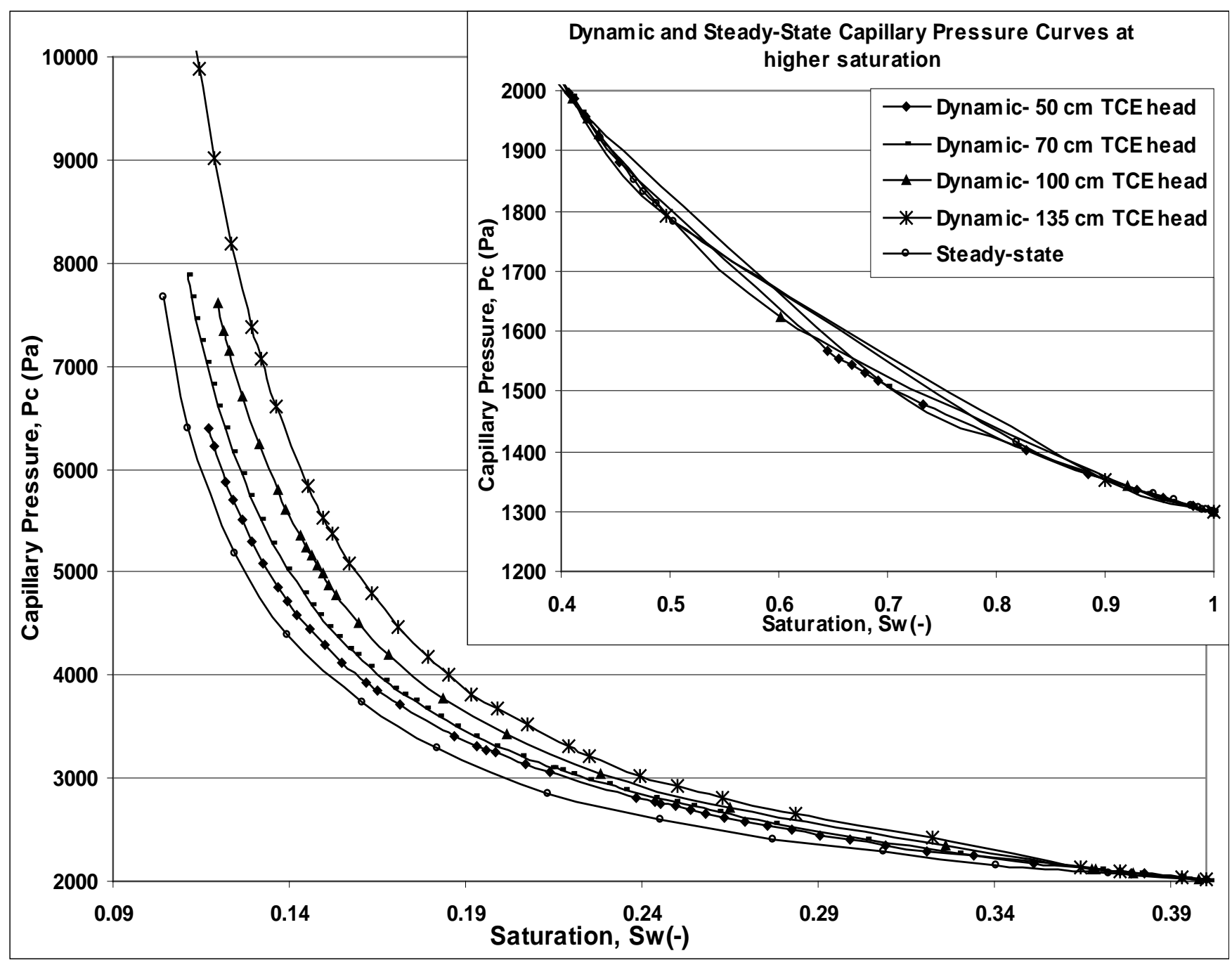

Figure 2. Steady-state and Dynamic Capillary Pressure-Saturation Curves for 3D Fine Sand Domain (homogeneous). The box shows the curves at higher water saturation. 


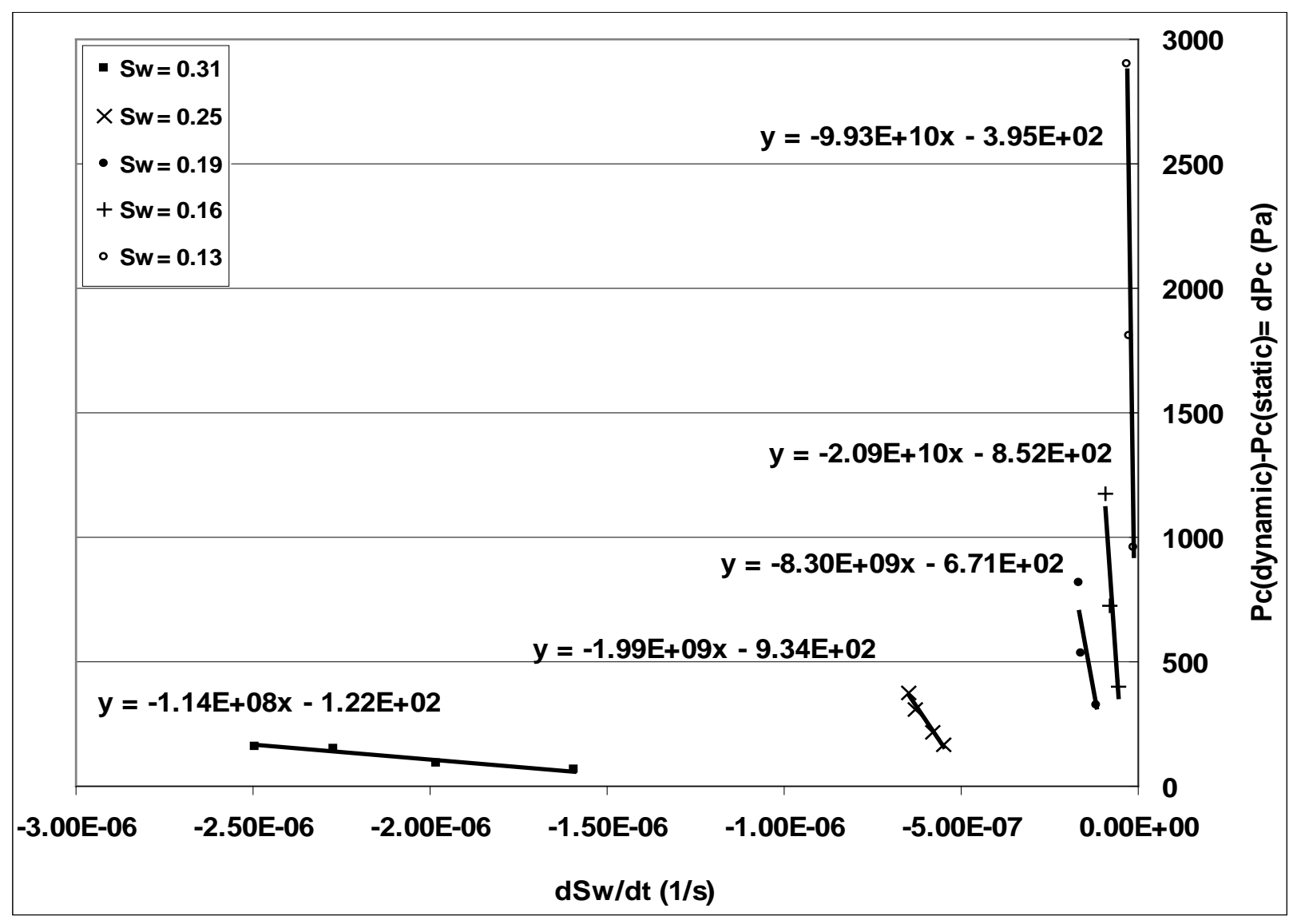

Figure 3(a) 


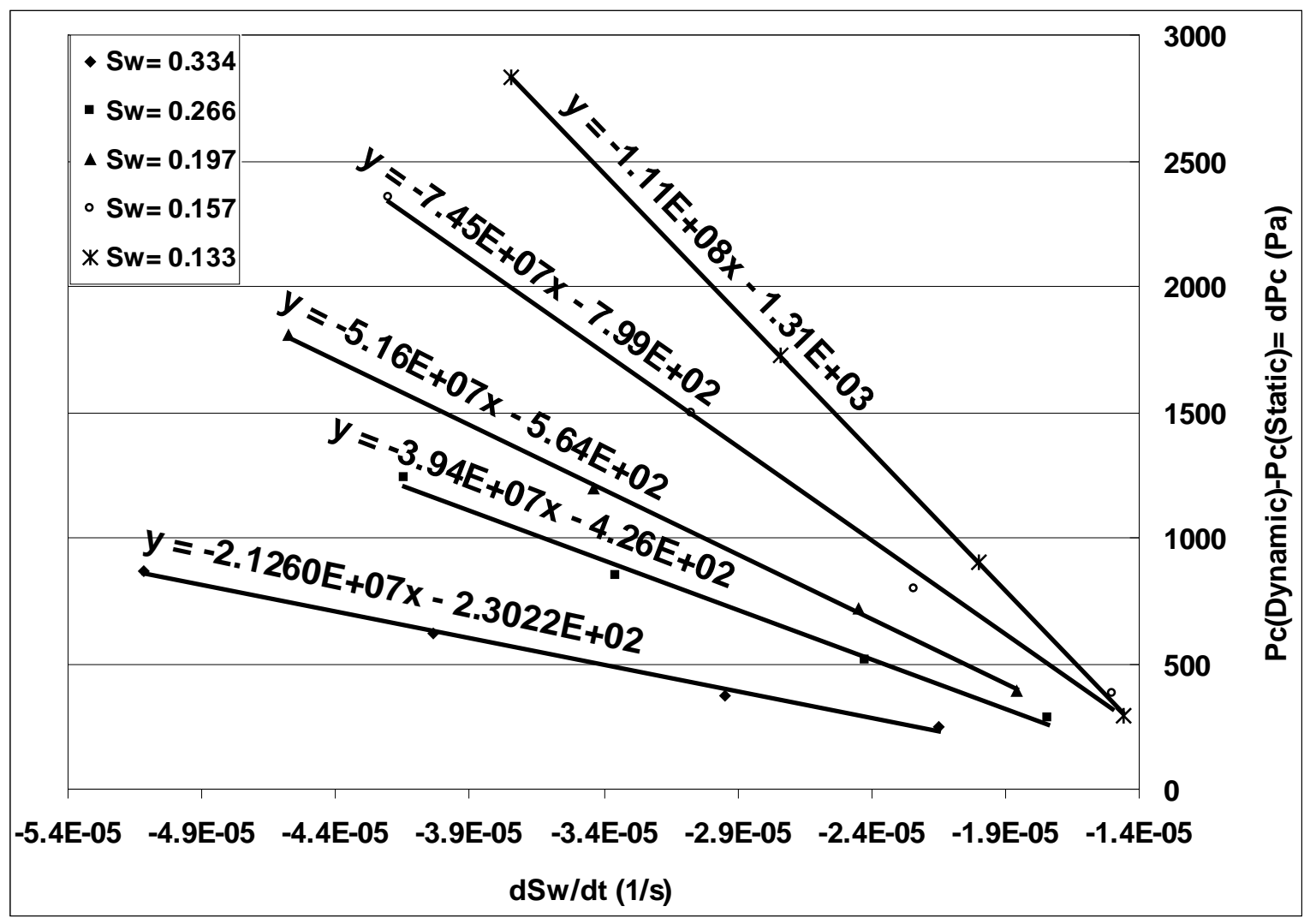

Figure 3(b) 


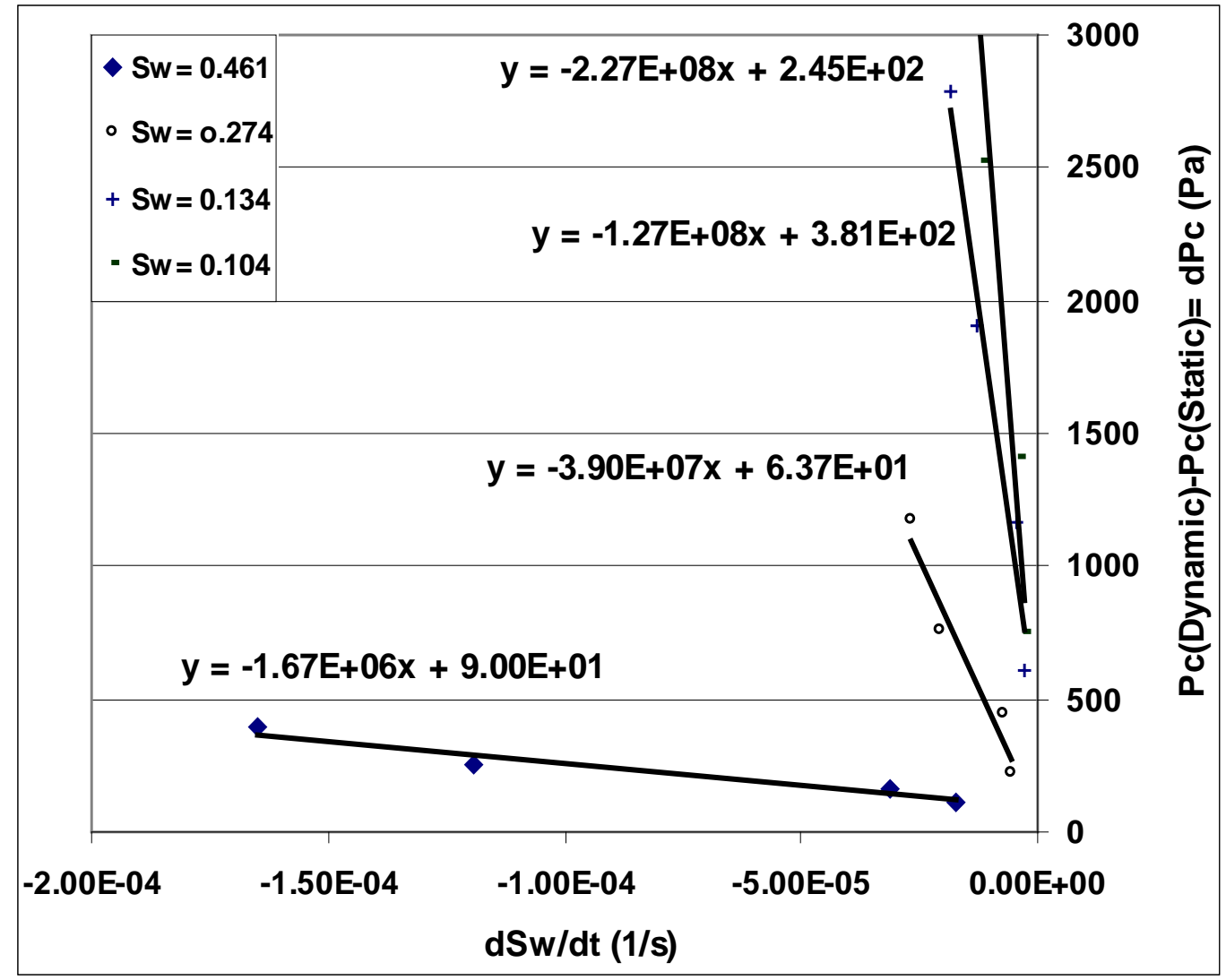

Figure 3(c) 


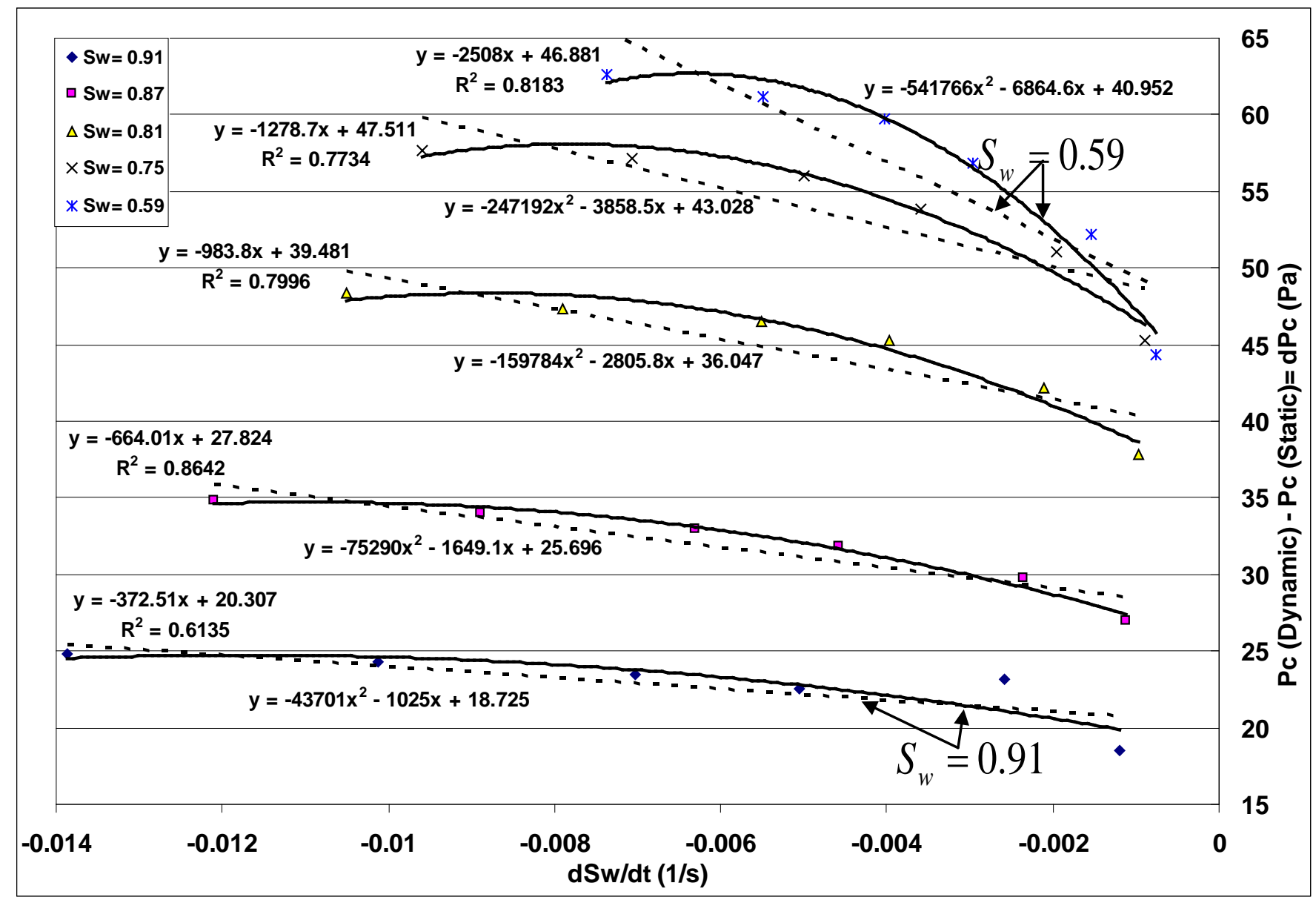

Figure 3(d)

Figure 3(a-d). Dynamic coefficient for (a) fine sand, (b) coarse sand, (c) heterogeneous sample with intensity of heterogeneity ( $\omega$ ) of 0.207 and, (d) fine sand at high saturations values. All simulations are carried out for 3D cylindrical samples (see, Figures 1 and 5). 


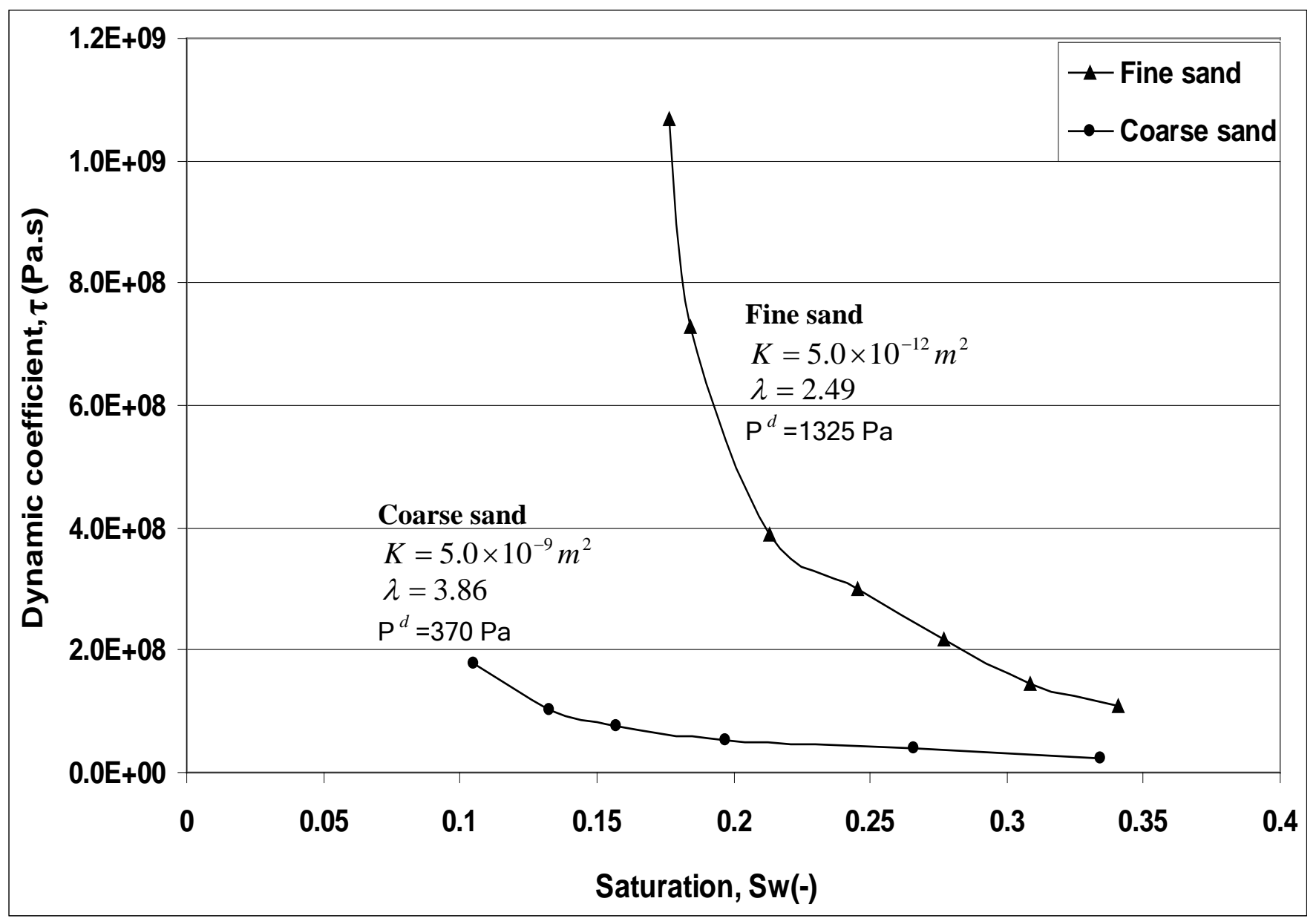

Figure 4. Pore size distribution $(\lambda)$ and permeability $(K)$ effects on dynamic coefficient $(\tau)$ value as a function of average saturation in a homogeneous cylindrical column consisting of either fine or coarse sand. 


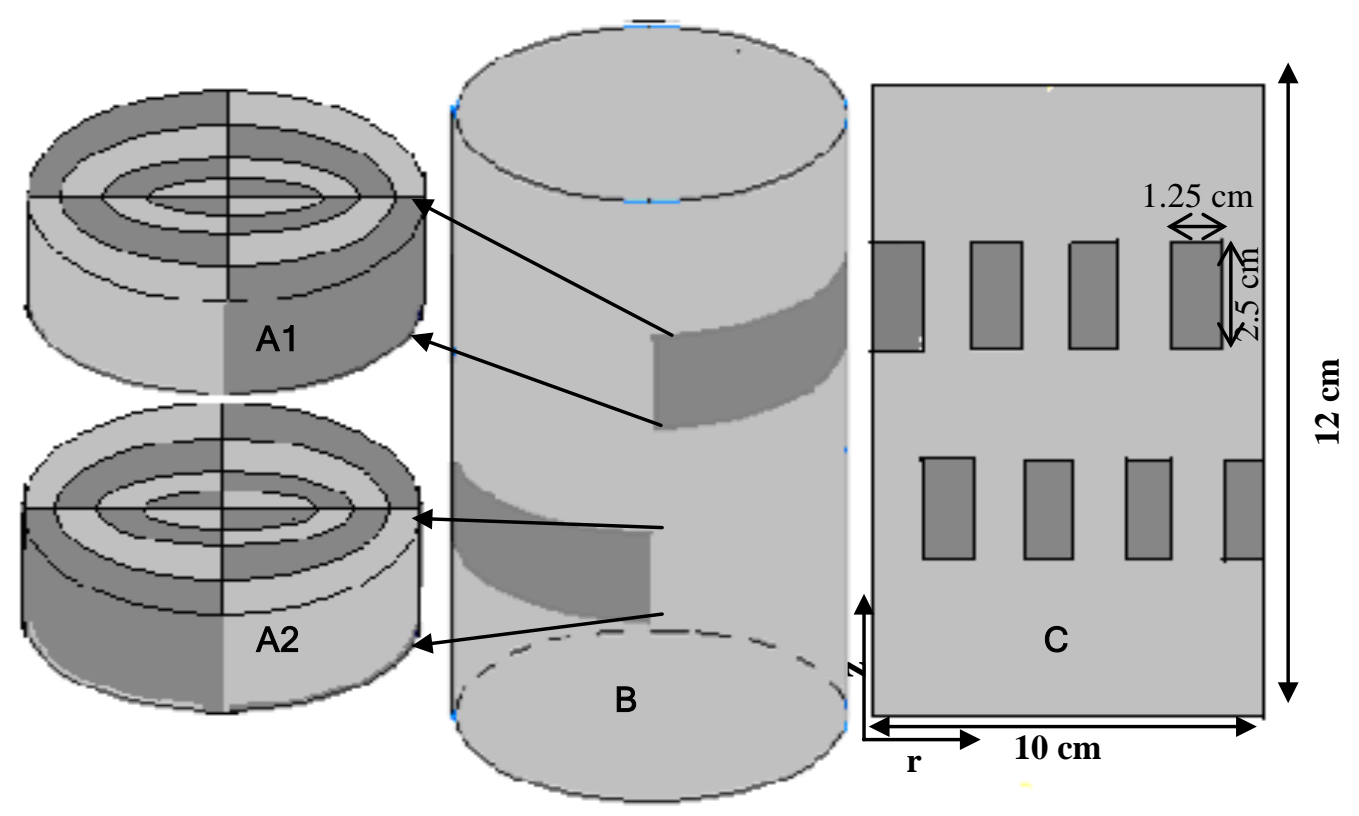

(a)

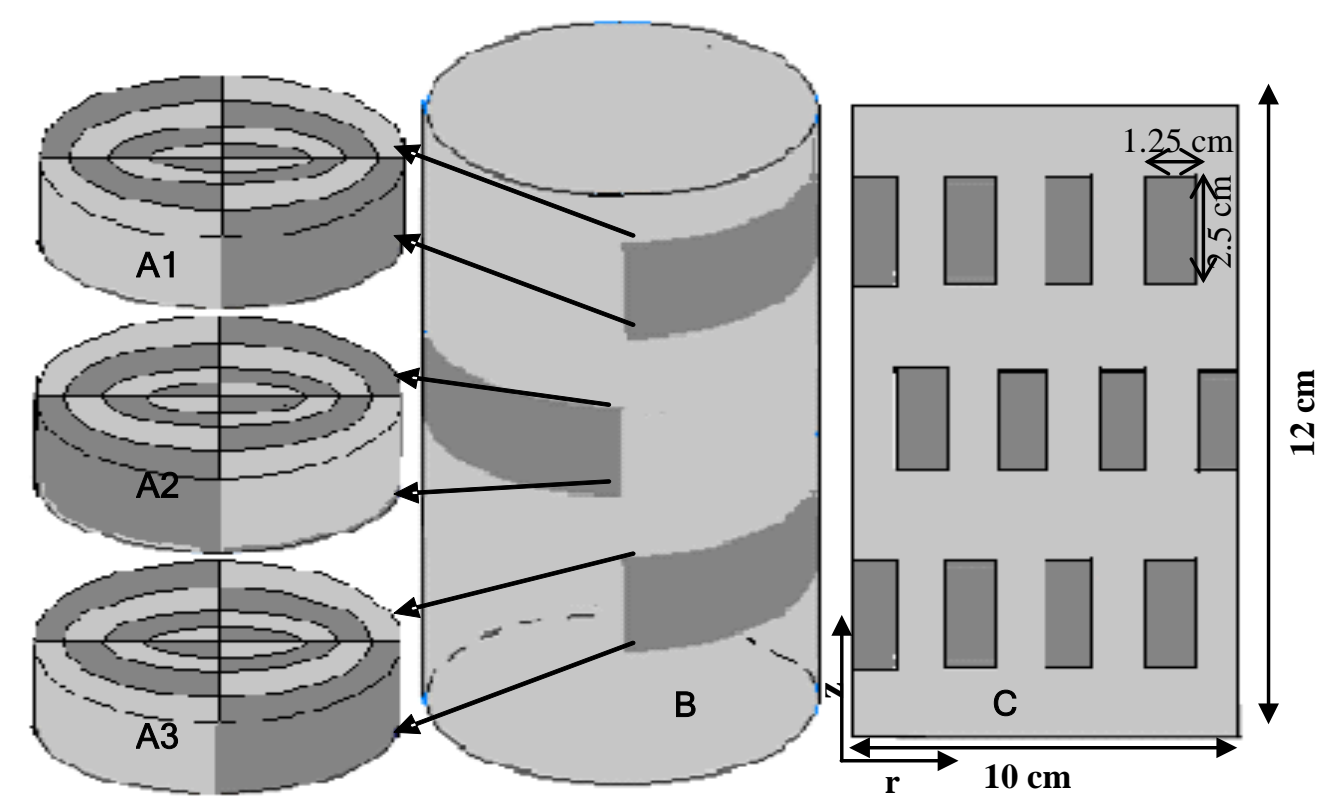

(b)

Figure 5 (a-b). 3D cylindrical domains for two intensities $(\omega)$ and distributions of microheterogeneities in the form of sub-sample scale fine sand blocks (dark grey regions) embedded in coarse sand background: (a) $\omega=0.207$ and (b) $\omega=0.372$.

(A1, A2, and A3) $\mathrm{r}-\Theta$ sections of heterogeneity distribution

(B) 3D heterogeneous sample displayed in cylindrical domain

(C) 2D rectangular (r-Z) section of heterogeneity distribution in cylindrical domain 


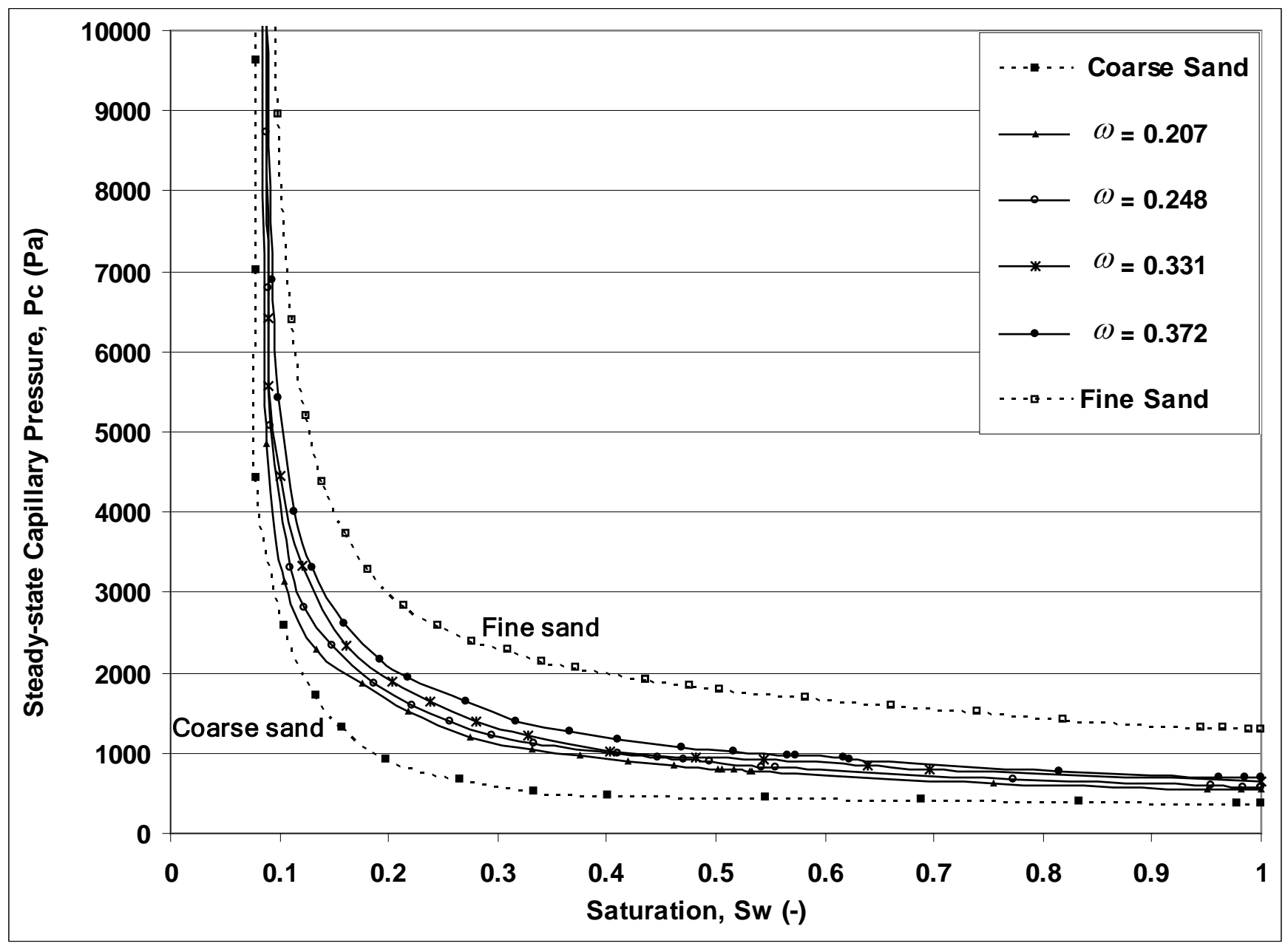

Figure 6(a) 


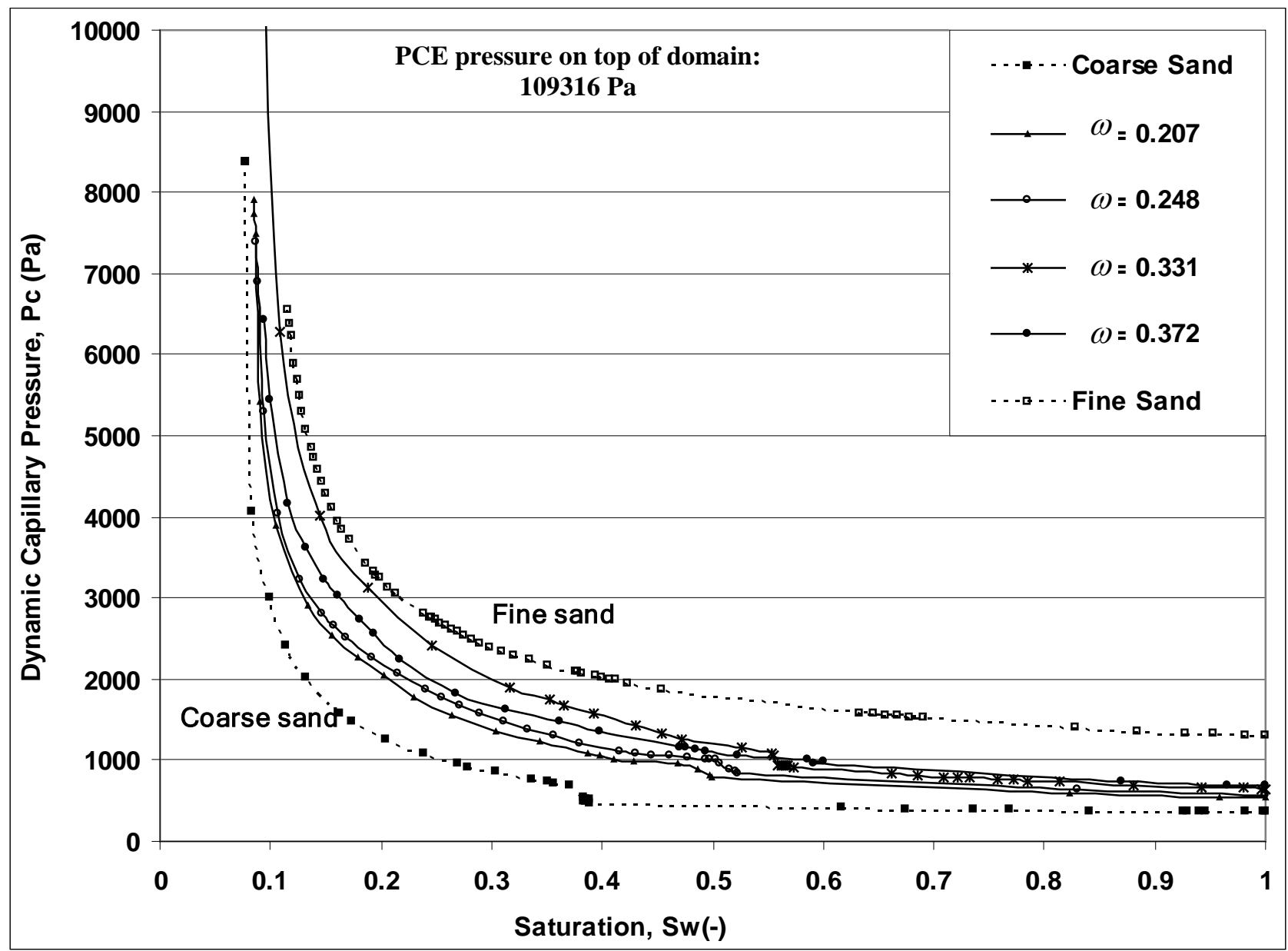

Figure 6(b) 


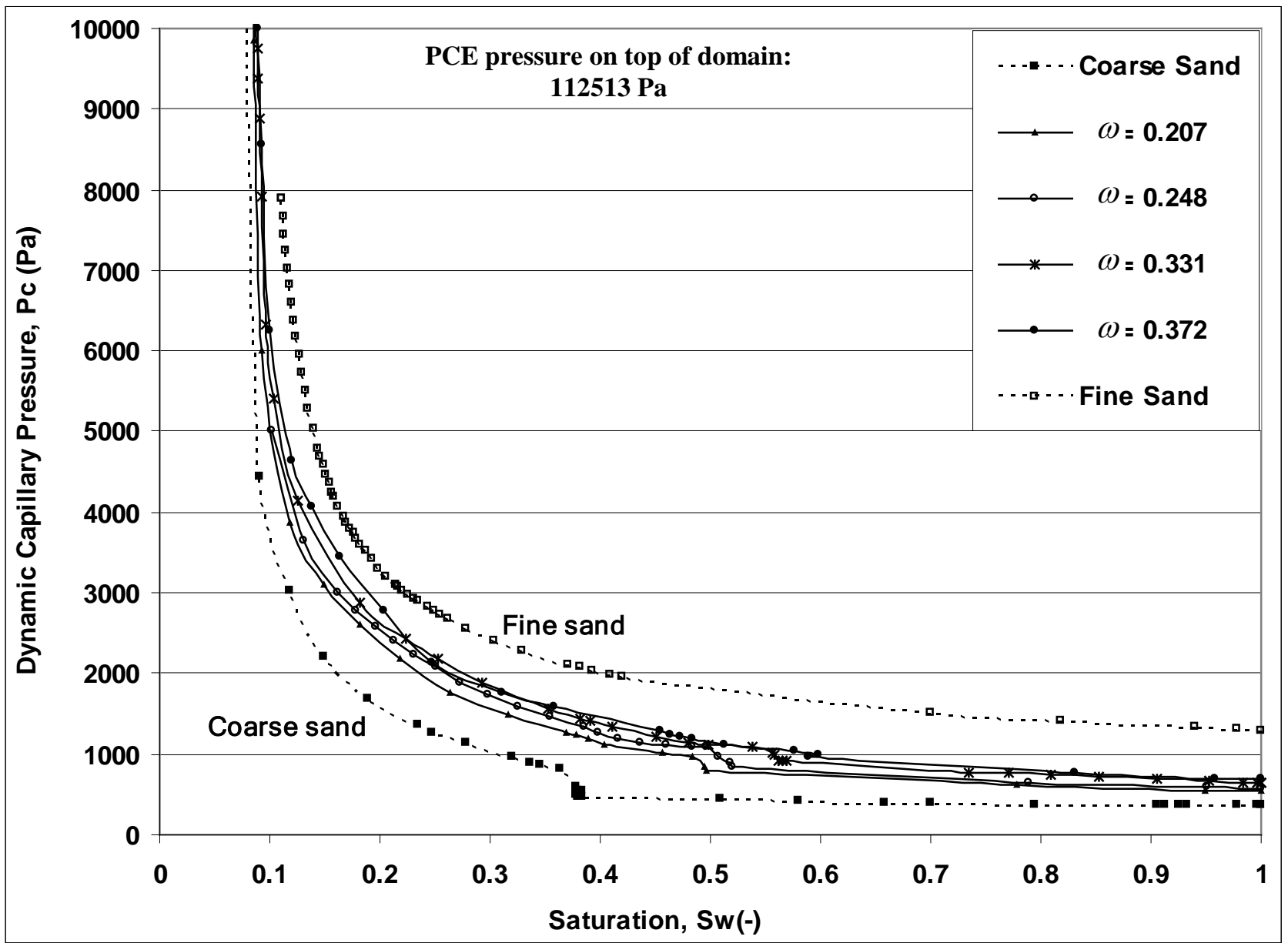

Figure 6(c) 


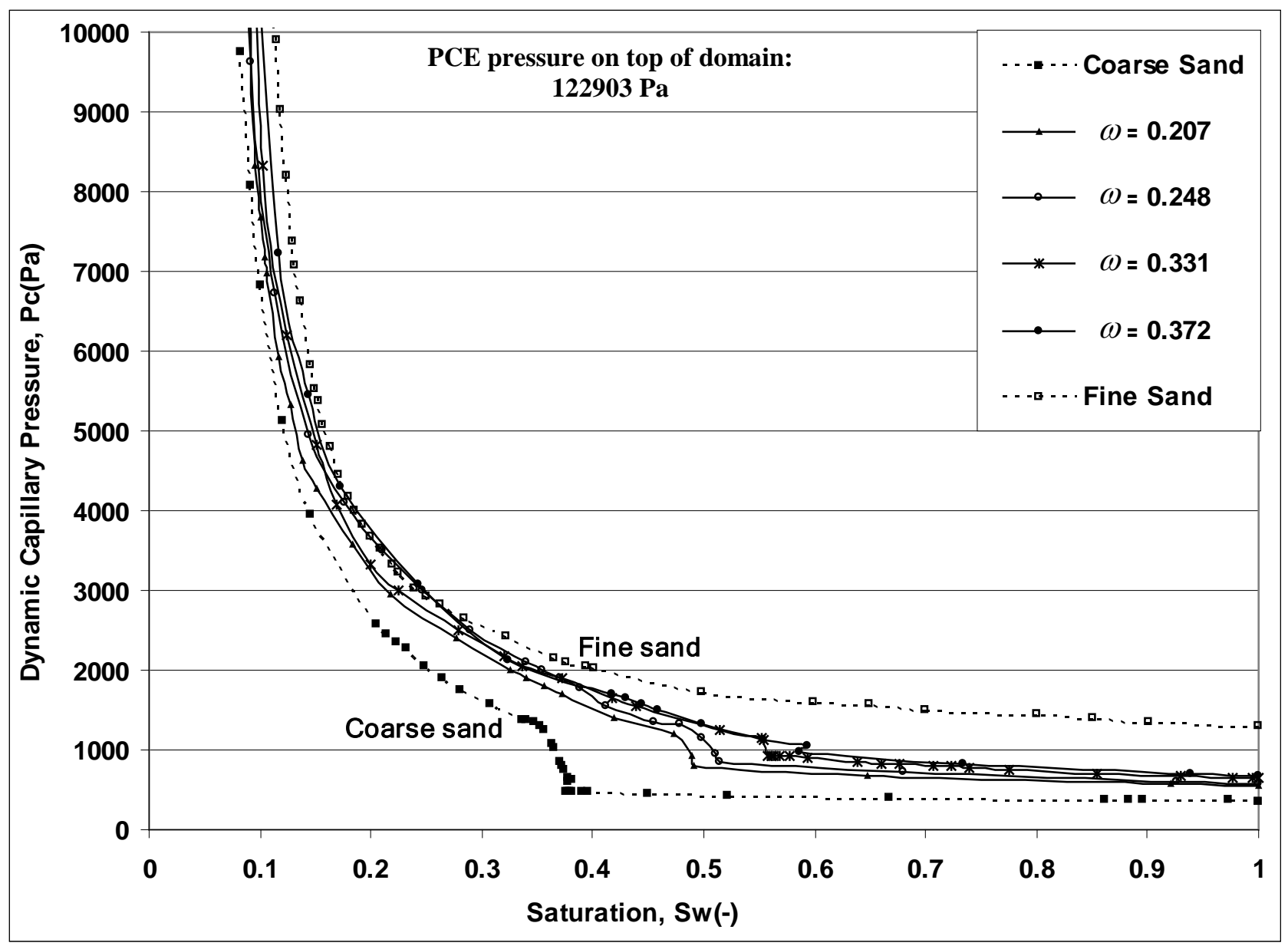

Figure 6(d)

Figure 6(a-d). Micro-Heterogeneity effects on (a) Steady-State and, Dynamic $\mathrm{P}^{\mathrm{c}}$-S curves for PCE pressure of (b) $109316 \mathrm{~Pa}$, (c) $112513 \mathrm{~Pa}$, and (d) $122903 \mathrm{~Pa}$ applied at the top of a cylindrical domain with various intensities of heterogeneity ( $\omega$ ). All results are for downward flow of PCE in cylindrical domains (3D). 


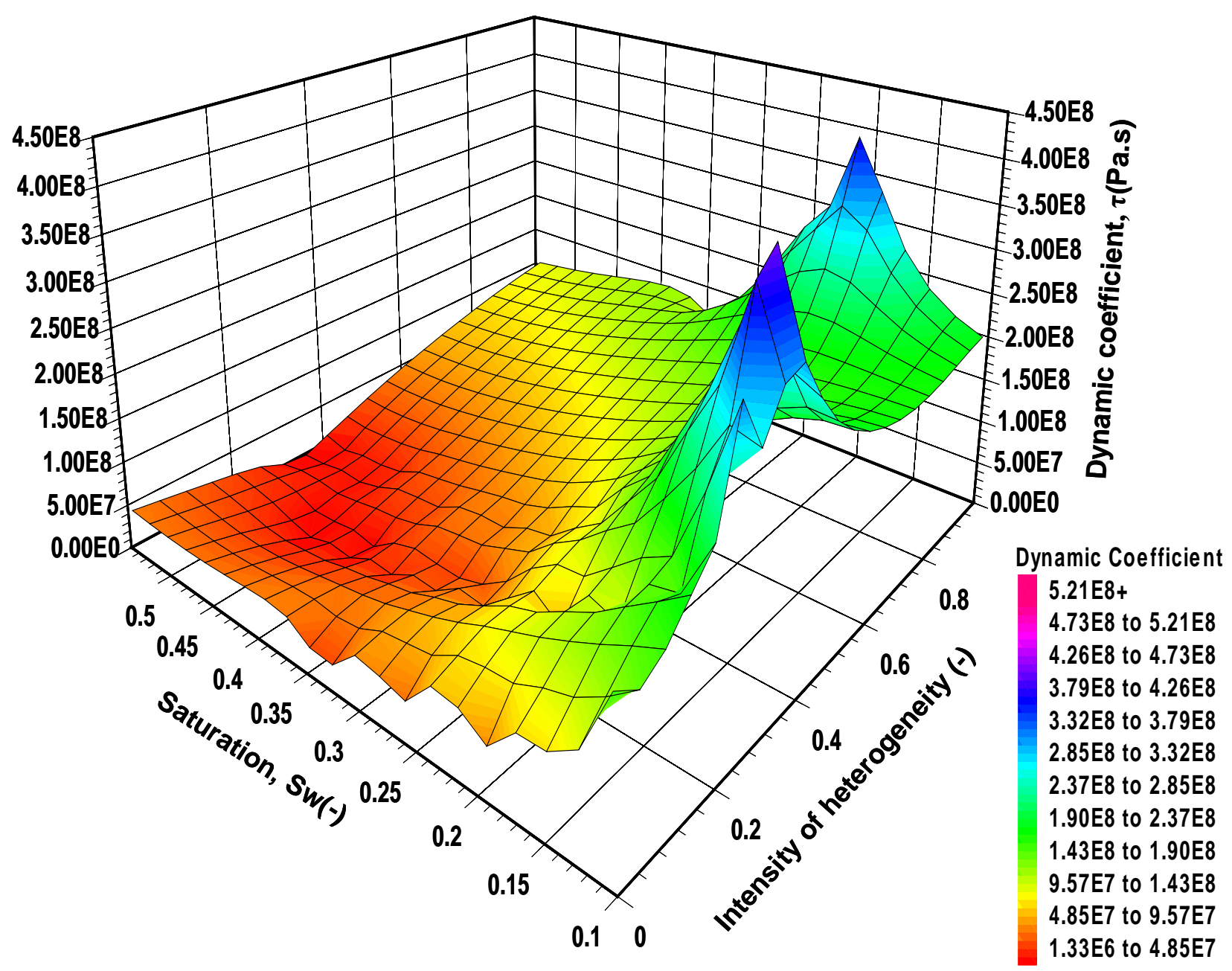

Figure 7. The effect of intensity of heterogeneity ( $\omega)$ on dynamic coefficient $(\tau)$. The value of $\tau$ is calculated for a 3D cylindrical domain (ID= $10 \mathrm{~cm}$ and height $=12 \mathrm{~cm}$ ) composed of either fine sand $(\omega=1)$ or coarse sand $(\omega=0)$ for homogeneous domains and fine sand lenses imbedded in coarse sand for heterogeneous domains. 


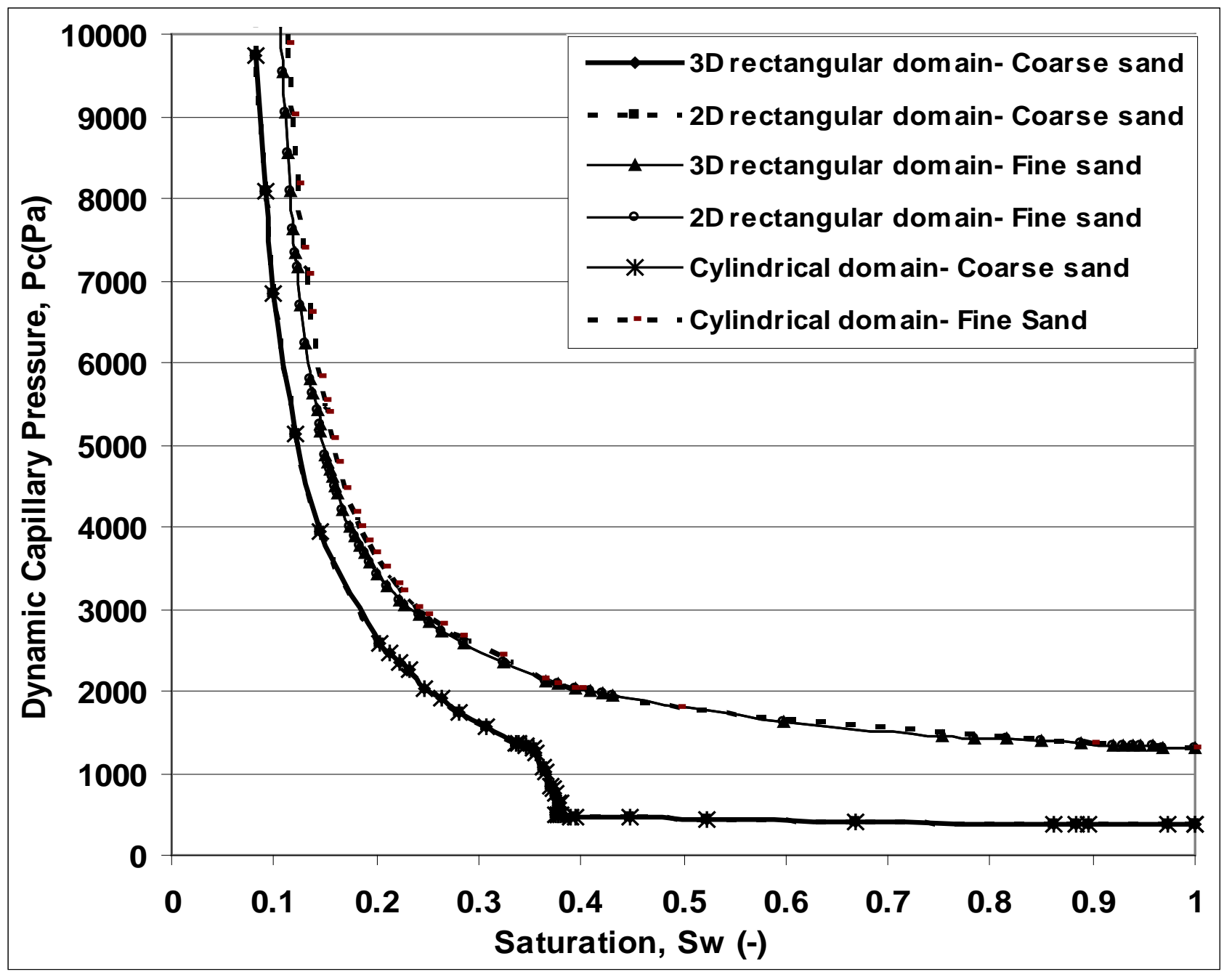

Figure 8(a) 


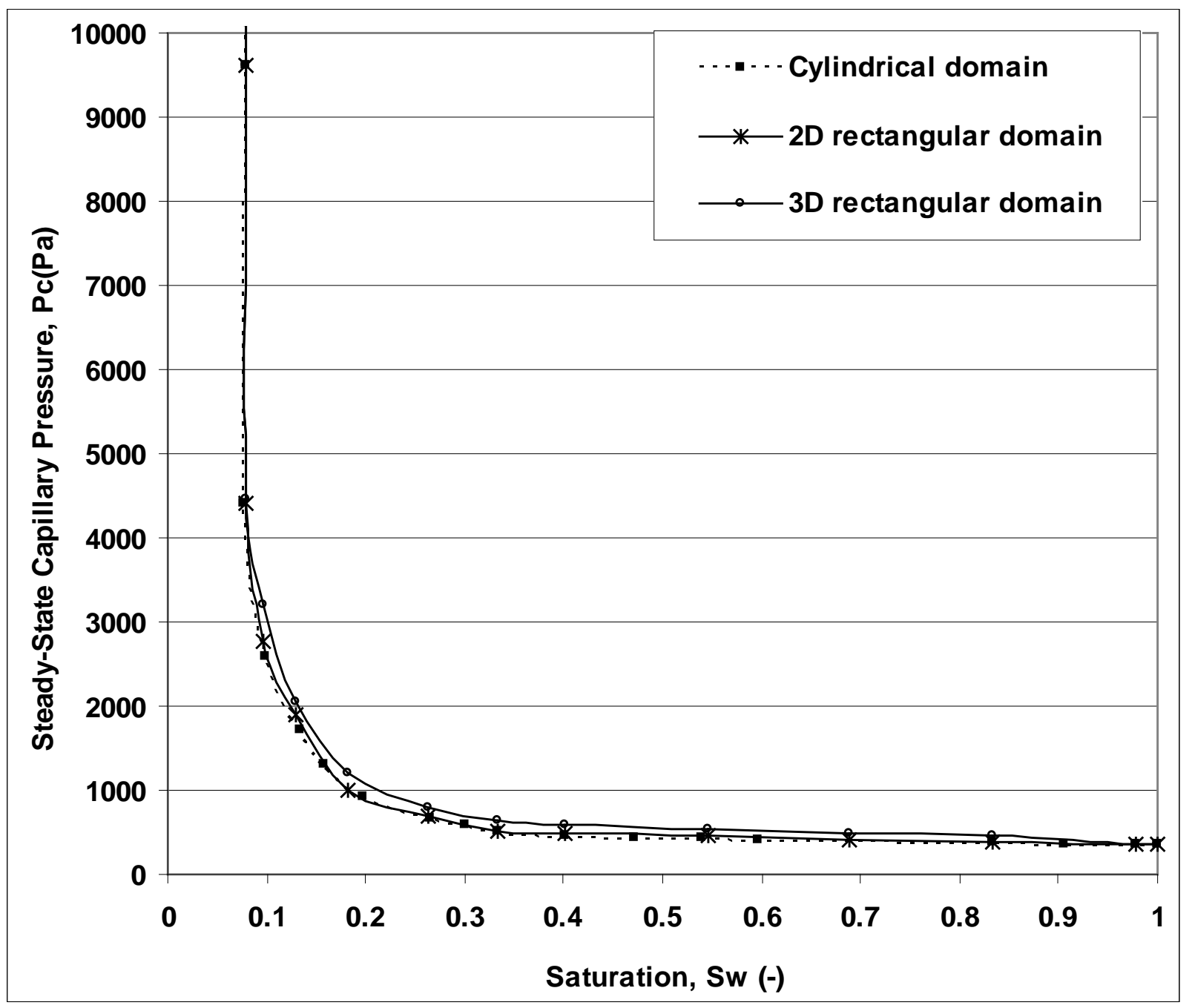

Figure 8(b)

Figure 8. (a) Effective dynamic capillary pressure curves for coarse and fine sand domain (b) Effective steadystate capillary pressure curves for coarse sand in different domain geometries. For fine sand the same trends are observed. 


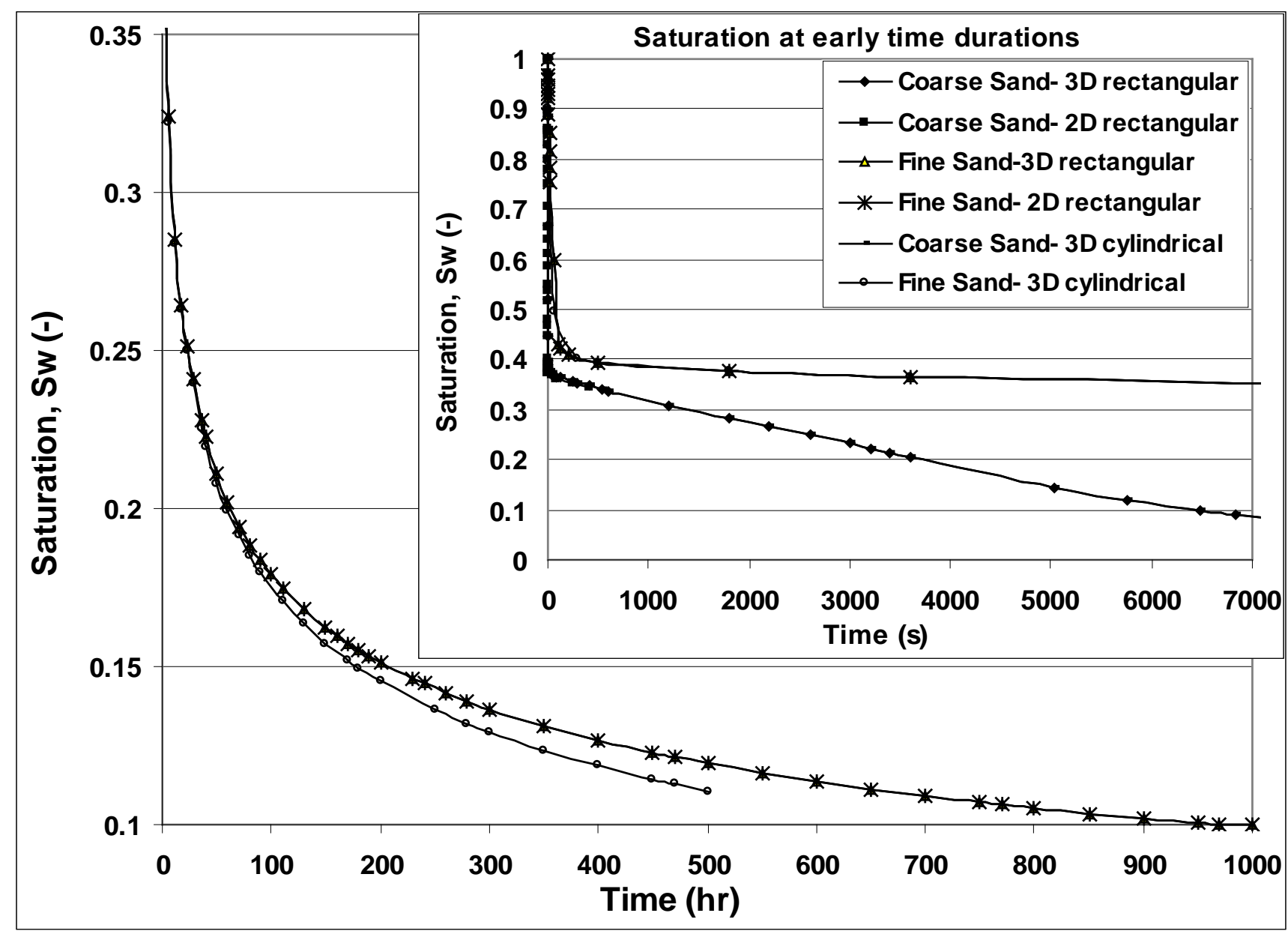

Figure 9. Saturation profile for coarse sand (in the box) and fine sand considering different domain geometries under dynamic flow condition. Saturation decrease rapidly in coarse sand sample (in less than 2 hours) because of high velocity fluid flow in large pore spaces and fast displacement of water by PCE in vertically downward flow. 


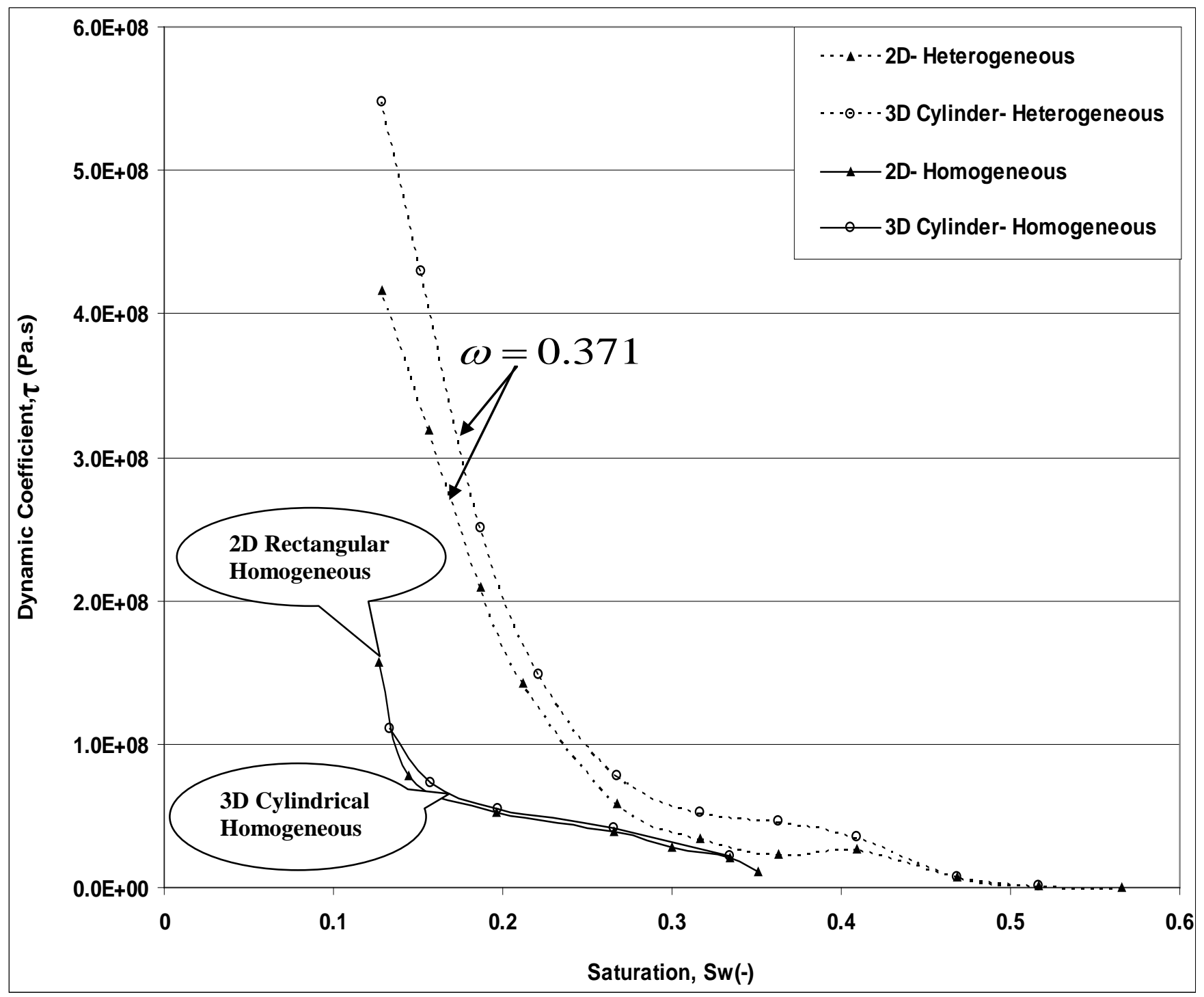

Figure 10. Domain geometry effects on dynamic coefficient. Geometry slightly affects the dynamic coefficient ( $\tau$ ) for flow in homogeneous coarse sand and heterogeneous domains for the geometry and dimensions shown in Figure 1. 


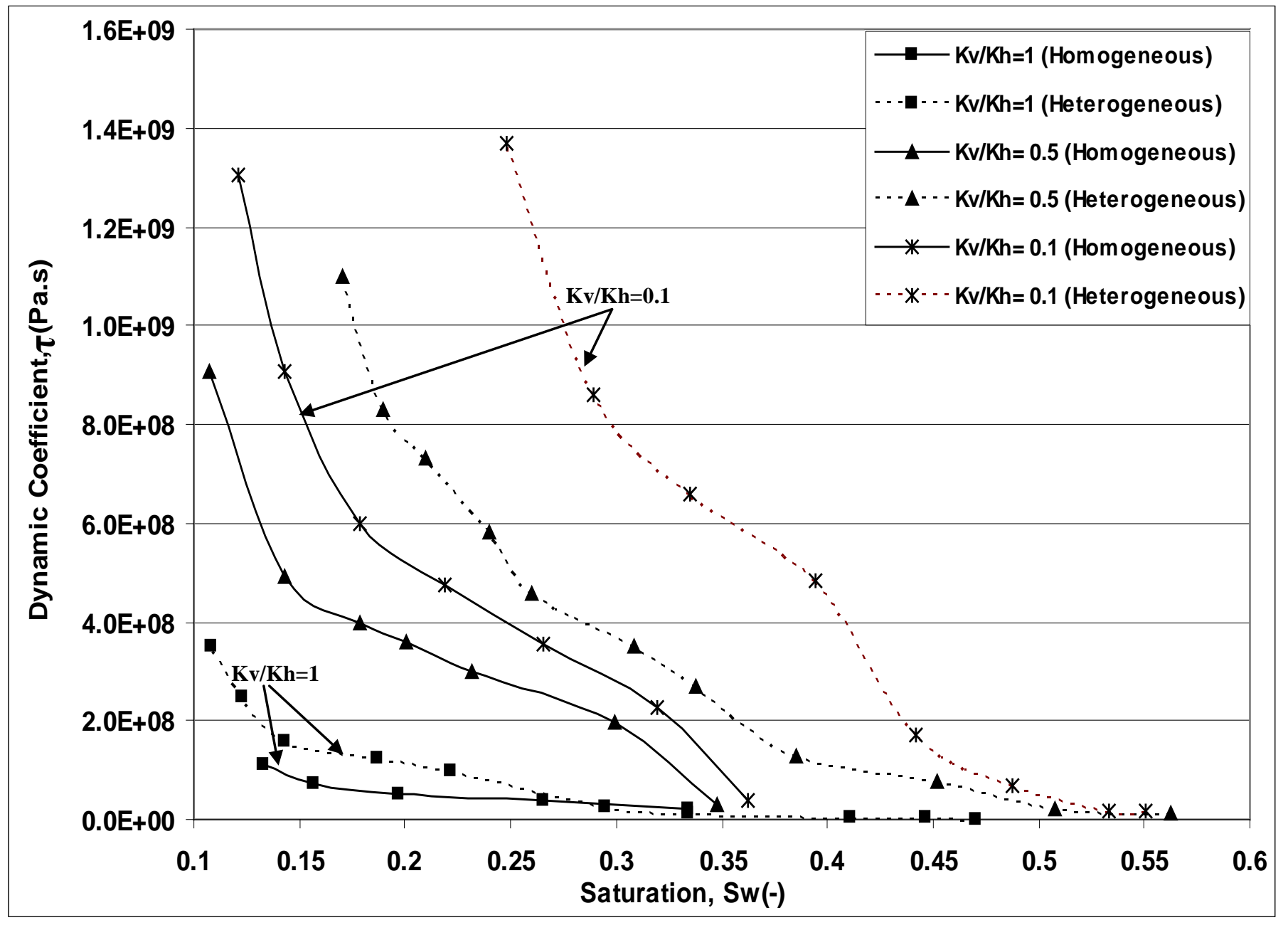

Figure 11. Effects of permeability anisotropy on dynamic coefficient for homogeneous (solid lines) and heterogeneous (dashed lines) domain with intensity of heterogeneity $(\omega)=0.207$. Anisotropy has been defines as the ratio of vertical permeability $\left(K_{v}=5.0 \times 10^{-9}\right)$ to the horizontal permeability $\left(K_{v} / K_{h}\right)$ where horizontal permeability remains the same in all directions (angles). 


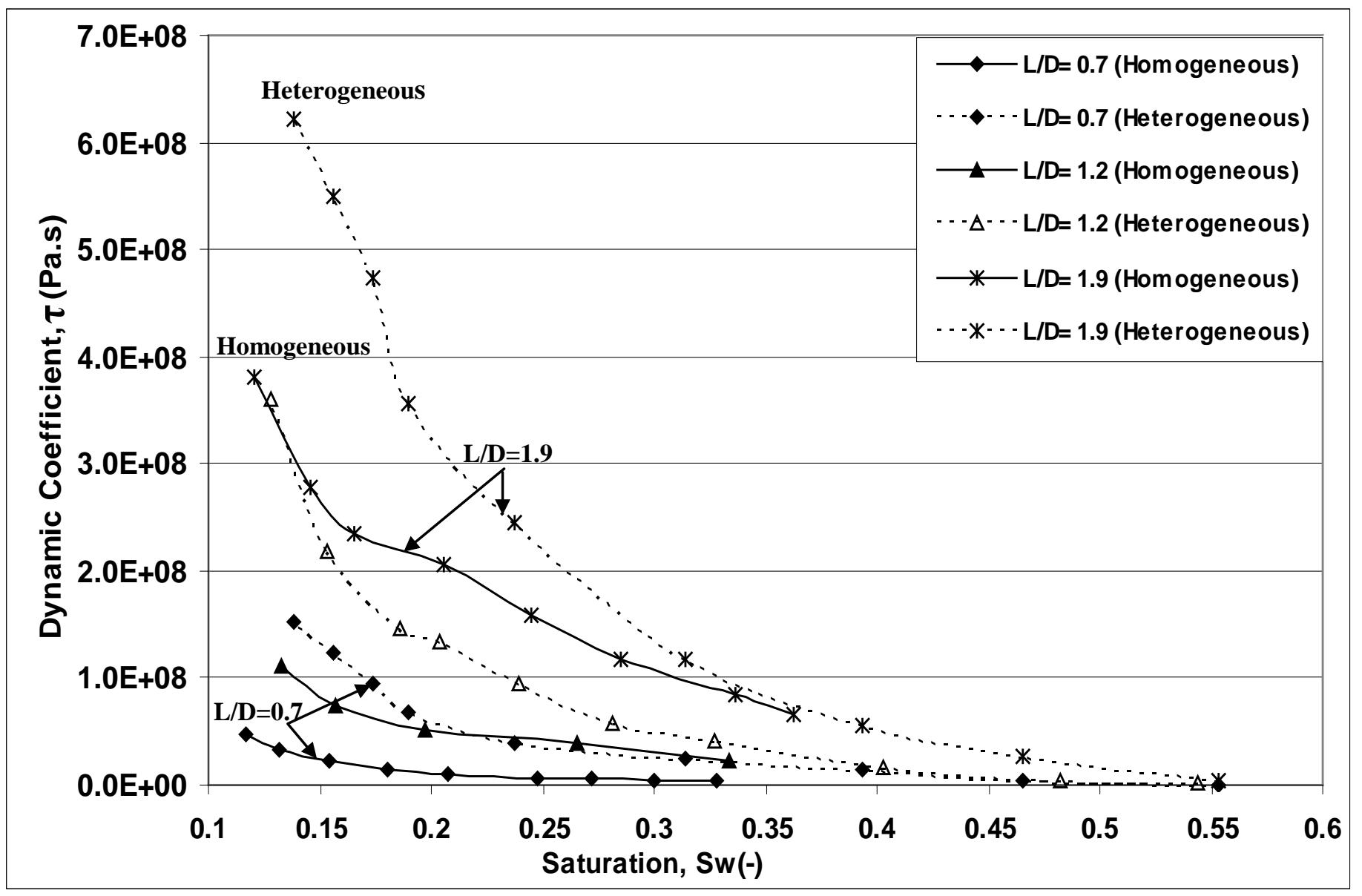

Figure 12. Effect of aspect ratio on dynamic coefficient for homogeneous and heterogeneous domains with intensity of heterogeneity $(\omega)=0.331$. Aspect ratio for cylindrical domain has been defined as Length/Diameter (L/D). In all cases the diameter (D) is kept constant while the length (L) is varied to obtain various $\mathrm{L} / \mathrm{D}$ ratios. 